\title{
Wireless Sensor Networks in Smart Structural Technologies
}

\author{
Yang Wang 1 and Kincho H. Law ${ }^{2}$ \\ ${ }^{1}$ Georgia Institute of Technology, Atlanta, Georgia, \\ 2Stanford University, Stanford, California,
}

USA

\section{Introduction}

Recent advances in wireless communication, as well as embedded computing, have opened many new exciting opportunities for wireless sensor networks. Miniature and low-cost wireless sensors are expected to become available in the next decade, offering countless possibilities for a wide range of applications. Among them is smart structural technology, an active research domain that holds significant promise for enhancing infrastructure management and safety. A smart structure refers to a specially equipped structure (e.g. buildings, bridges, dams, etc.) that can monitor and react to surrounding environment and the structure's own conditions, in a pre-designed and beneficial manner.

Smart structural technology encompasses at least two major fields, i.e. structural health monitoring and structural control. A structural health monitoring (SHM) system measures structural responses and predicts, identifies, and locates the onset of structural damage, e.g. due to deterioration or hazardous events. Structural sensors, such as micro-electromechanical system (MEMS) accelerometers, metal foil strain gages, fiber optic strain sensors, among others, have been developed and employed to collect important information about civil structures that could be used to infer the safety conditions of the structure (Farrar, et al. 2003, Sohn, et al. 2003, Chang 2009). On the other hand, structural control technology aims to mitigate adverse effects due to excessive dynamic loads (Yao 1972, Soong 1990, Housner, et al. 1997, Spencer and Nagarajaiah 2003).

Structural monitoring and control both involve acquiring response data in real time. In order to transmit real-time data, coaxial cables are normally employed as the primary communication link. Cable installation is labor intensive and time consuming, and can cost as much as $\$ 5,000$ US dollars per communication channel (Çelebi 2002). To eradicate the high cost incurred by the use of cables, wireless systems could serve as a viable alternative (Straser and Kiremidjian 1998). Wireless communication standards, such as Bluetooth (IEEE 802.15.1), Zigbee (IEEE 802.15.4), Wi-Fi (IEEE 802.11b), are now mature and reliable technologies widely adopted in many industrial applications (Cooklev 2004). Potential applications of wireless technologies in structural health monitoring have been explored by a number of researchers, as reviewed by Lynch and Loh (2006). By incorporating a control interface, wireless sensors have also been extended to potentially command control devices for structural control applications (Wang, et al. 2007b). 
Compared to cable-based systems, wireless structural monitoring and control systems have a unique set of advantages and technical challenges. Besides the desire for portable longlasting energy sources, such as batteries, reliable data communication is a key issue for implementation. The purpose of this chapter is to review the important issues and metrics for adopting wireless sensor networks in smart structural systems. In a structural health monitoring system, sensors are typically deployed in a passive manner, primarily for measuring structural responses. Structural control systems, on the other hand, need to respond in real time to mitigate excess dynamic response of structures. Typical feedback control systems require real-time information and measurements to instantly determine control decisions. Although structural monitoring and control applications pose different needs and requirements, efficient information flow plays a key and critical role in both implementations. For example, the transmission latency and limited bandwidth of wireless devices can impede real-time operations as required by control or monitoring systems. In addition, communication in a wireless network is inherently less reliable than that in cablebased systems, particularly when node-to-node communication range lengthens. These information constraints, including bandwidth, latency, range, and reliability, need to be considered carefully using an integrated system approach and pose many challenges in the selection of hardware technologies and the design of software/algorithmic strategies.

The chapter adopts a previously designed wireless structural monitoring and control system as an example to discuss various intriguing research challenges (Wang, et al. 2005, Wang 2007). The system contains wireless sensing and control units that can be used for both wireless structural health monitoring and real-time feedback structural control. Modularized software is designed for the wireless units, so that application programs can be conveniently embedded into the units. The architectural details of the wireless structural monitoring and control system are presented. For different structural applications, including health monitoring and control, special communication protocols have been designed to efficiently manage the information flow among the wireless units. Finally, laboratory and field validation tests have been conducted to assess the performance of the prototype wireless structural monitoring and control system.

\section{Design and implementation of a wireless sensing and control unit}

Sensing and control units are the fundamental components of a wireless monitoring and control system. The prototype wireless unit is designed in such a way that the unit can serve as either a sensing unit (i.e. a unit that collects data from sensors and wirelessly transmits the data), a control unit (i.e. a unit that calculates optimal control decisions and commands control devices), or a unit for both sensing and control. Fig. 1 shows the functional diagram of the prototype wireless sensing and control unit. The wireless sensing unit shown in the top part of Fig. 1 serves as the core component, with which off-board modules for signal conditioning and signal generation can be easily incorporated.

\subsection{Hardware and software of the wireless sensing and control unit}

The wireless sensing unit consists of three functional modules: sensor signal digitization, computational core, and wireless communication. The sensing interface converts analog sensor signals into digital data, which is then transferred to the computational core through a high-speed Serial Peripheral Interface (SPI) port. Besides a low-power 8-bit Atmel ATmega128 microcontroller, external Static Random Access Memory (SRAM) is integrated 


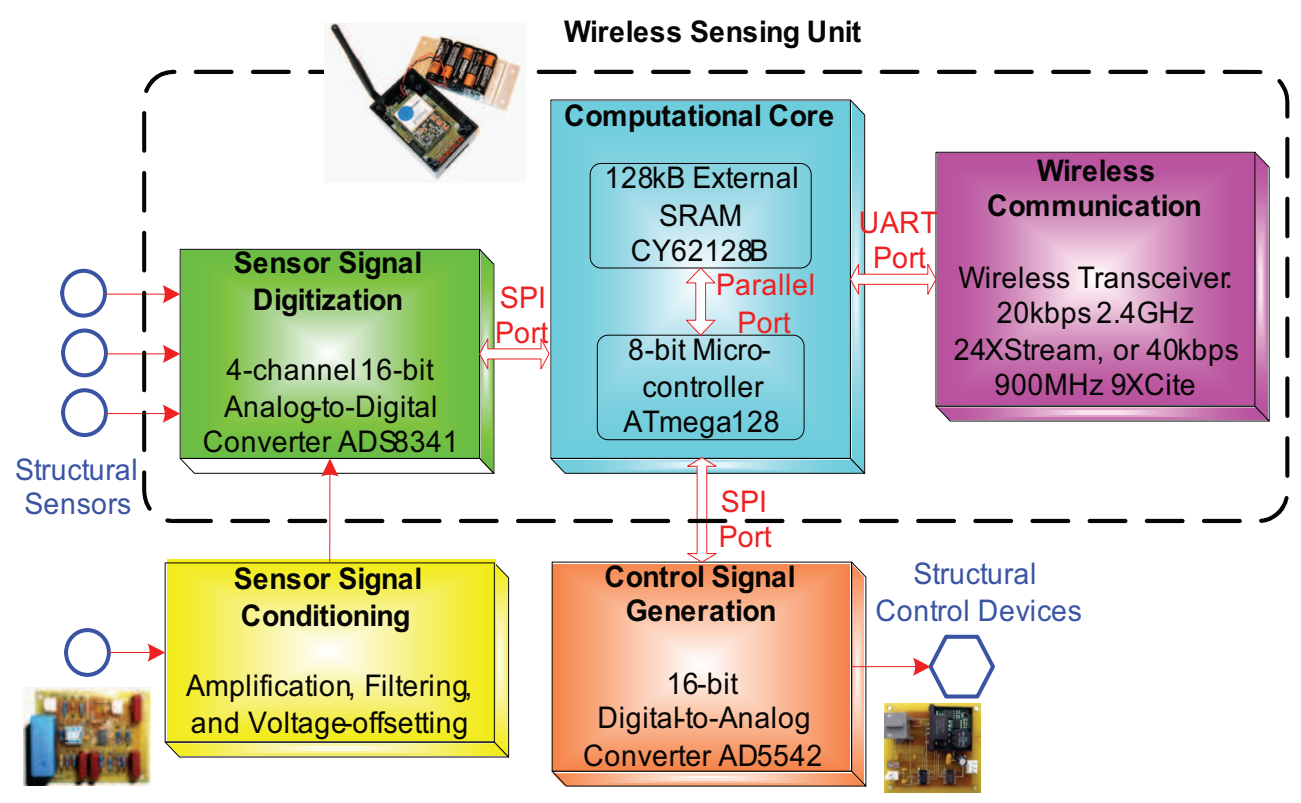

Fig. 1. Functional diagram detailing the hardware design of the wireless sensing unit. Additional off-board modules can be interfaced to the wireless sensing unit to condition sensor signals and issue control commands

with the computational core to accommodate local data storage and analysis. The computational core communicates with a wireless transceiver (24XStream or 9XCite models currently provided by Digi International) through a Universal Asynchronous Receiver and Transmitter (UART) interface. The auxiliary sensor signal conditioning module assists in amplifying, filtering, and offsetting analog sensor signals prior to digitization. The auxiliary control signal generation module offers an interface through which the wireless sensor can send analog control commands to structural control devices. Hardware design of the wireless unit and auxiliary modules have been described in details elsewhere (Wang, et al. 2005, Wang 2007, Wang, et al. 2007a). The key parameters of the prototype wireless sensing unit are summarized in Table 1. Peer-to-peer communication among wireless units is supported for collaborative data analysis.

In order to manage the hardware components in a wireless sensing unit, software modules are implemented and embedded in the ATmega128 microcontroller. For the ATmega128 microcontroller, software can be written in a high-level programming language, such as $\mathrm{C}$, compiled into binary instructions, and loaded into the non-volatile flash memory of the microcontroller. When the wireless unit is powered on for normal operation, the microcontroller automatically starts executing the embedded instructions. The software design of the wireless sensing and control units follows the hierarchical structure as shown in Fig. 2. At the bottom level are the software modules that manage the basic peripherals of the microcontroller. The middle layer consists of software modules that manage other onboard hardware components. Specific software modules for structural health monitoring and control are implemented in the top level application layer. 


\begin{tabular}{|c|c|c|}
\hline Design Parameter & \multicolumn{2}{|c|}{ Specification } \\
\hline Computing Core & & \\
\hline Microcontroller & \multicolumn{2}{|c|}{$\begin{array}{l}\text { 8-bit RISC }{ }^{1} \text { architecture, up to } 16 \mathrm{MIPS}^{2} \text { throughput at } \\
\qquad 16 \mathrm{MHz}\end{array}$} \\
\hline Flash Memory & \multicolumn{2}{|c|}{$128 \mathrm{~K}$ bytes } \\
\hline Internal SRAM ${ }^{3}$ & \multicolumn{2}{|c|}{$4 \mathrm{~K}$ bytes } \\
\hline External SRAM & \multicolumn{2}{|c|}{$128 \mathrm{~K}$ bytes } \\
\hline EEPROM 4 & \multicolumn{2}{|c|}{$4 \mathrm{~K}$ bytes } \\
\hline Power Consumption & \multicolumn{2}{|c|}{$30 \mathrm{~mA}$ active, $55 \mu \mathrm{A}$ standby } \\
\hline Wireless Transmission & 9XCite & 24XStream \\
\hline Operating Frequency & ISM 902-928 MHz & ISM 2.4000 - $2.4835 \mathrm{GHz}$ \\
\hline Data Transfer Rate & $38.4 \mathrm{kbps}$ & $19.2 \mathrm{kbps}$ \\
\hline $\begin{array}{l}\text { Communication } \\
\text { Range }\end{array}$ & $\begin{array}{l}\text { Up to } 300^{\prime}(90 \mathrm{~m}) \text { indoor, } \\
1000^{\prime}(300 \mathrm{~m}) \text { at line-of-sight }\end{array}$ & $\begin{array}{l}\text { Up to } 600^{\prime}(180 \mathrm{~m}) \text { indoor, } 3 \\
\text { miles }(5 \mathrm{~km}) \text { at line-of-sight }\end{array}$ \\
\hline Power Consumption & $\begin{array}{l}55 \mathrm{~mA} \text { transmitting, } 35 \mathrm{~mA} \\
\text { receiving, } 20 \mu \mathrm{A} \text { standby }\end{array}$ & $\begin{array}{l}150 \mathrm{~mA} \text { transmitting, } 80 \mathrm{~mA} \\
\text { receiving, } 26 \mu \mathrm{A} \text { standby }\end{array}$ \\
\hline Sensing Interface & \multicolumn{2}{|c|}{4 channels, 16-bit, up to $100 \mathrm{kHz}$} \\
\hline Control Interface & \multicolumn{2}{|c|}{1 channel, 16-bit, up to $1 \mathrm{MHz}$} \\
\hline Physical Size & \multicolumn{2}{|c|}{$10.2 \mathrm{~cm} \times 6.5 \mathrm{~cm} \times 4.0 \mathrm{~cm}$} \\
\hline
\end{tabular}

Table 1. Key parameters of the wireless sensing unit

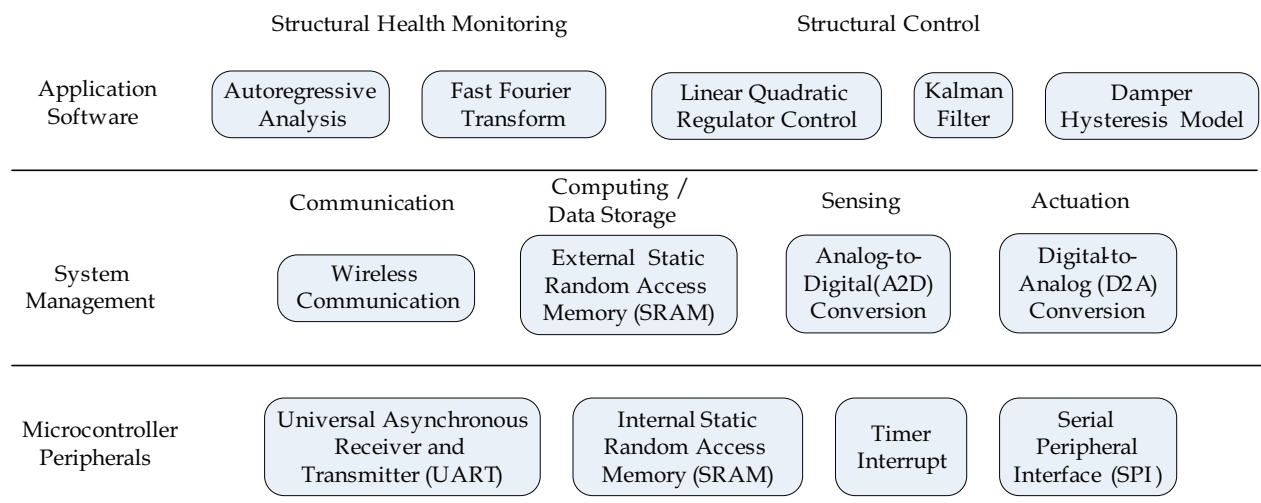

Fig. 2. Three-layer software architecture for the ATmega128 microcontroller in the wireless sensing and control unit

${ }^{1}$ RISC: reduced instruction set computer.

2 MIPS: million instructions per second.

${ }^{3}$ SRAM: static random access memory.

${ }^{4}$ EEPROM: electrically erasable programmable read-only memory. 
As shown in Fig. 2, the lowest level of the embedded software manages the peripherals of the ATmega128 microcontroller and serves as the fundamental modules to support the functions of other hardware components. Embedded modules include: timer interrupt functions, byte-by-byte communication through the UART and SPI ports, and internal memory management. The timer interrupt service is implemented to achieve a constant time step for sensor data sampling. The interrupt function is also a powerful feature that allows the software to momentarily pause an executing task (such as data processing or wireless communication) to sample data from the sensing interface according to a precise timing schedule. Immediately after servicing the sensing interface, the paused task is resumed and the program continues its execution. This timer interrupt feature is utilized to implement continuous data streaming from multiple wireless sensing units, where sensor data sampling has to occur at a constant sampling step amidst the execution of the wireless communication or data interrogation program. In effect, the software supports concurrency thereby allowing multiple software tasks to execute at the same time.

Building on top of the microcontroller peripherals are the software drivers that manage other hardware components in the wireless unit. Utilizing the UART peripheral, the wireless communication driver provides the following functions interfacing the microcontroller with the wireless transceiver: 1) reading or setting the radio parameters of the attached wireless transceiver; 2) sending or receiving data through the wireless transceiver; 3) implementing the state machine representing the wireless communication protocol. A driver module is implemented to manage the $128 \mathrm{kB}$ external Static Random Access Memory (SRAM). This module includes functions to enable and disable the external SRAM, as well as functions that allow access to the lower $64 \mathrm{kB}$ half or higher $64 \mathrm{kB}$ half of the memory chip. The other two hardware drivers, the A2D and the D2A modules, manage the interfaces with the structural sensors and control devices. The ATmega128 microcontroller provides only one SPI port, which is shared by both the A2D converter (ADS8341) for sensing and the D2A converter (AD5542) for control. The A2D module commands the ADS8341 to convert a 0 to $5 \mathrm{~V}$ analog sensor signal into a 16-bit integer. Knowing the sensitivity and offset of the sensor signal, the microcontroller can then compute a floating-point number quantifying the physical parameter being measured by the sensor. Conversely, the D2A module takes a floating-point number between $-5 \mathrm{~V}$ and $5 \mathrm{~V}$ as input, converts the number into a 16-bit integer, and pushes the integer to the AD5542 to output the corresponding control voltage signal.

Utilizing the hardware drivers for communication, computing, sensing, and control, software can be developed to support structural health monitoring and control applications. A number of engineering algorithms, such as Fast Fourier Transform (FFT), autoregressive (AR) analysis, linear quadratic regulator (LQR) control, and Kalman Filter, have been implemented and embedded in the wireless units. The ability to execute embedded application software allows the wireless sensing units to make and execute decisions. Onboard data processing also helps save energy resources (i.e. preserving limited battery power) by reducing wireless transmission of large amounts of raw sensor data. With the application software executing in the wireless unit, each unit acts as an autonomous node in a wireless monitoring and control network. This architecture of distributed sensing and control represents a new paradigm in structural health monitoring and control, as opposed to traditional centralized systems, where data are processed in a centralized location. 


\subsection{Communication constraints}

As noted in Table 1, the sensing unit is designed to support two wireless transceivers: 900$\mathrm{MHz}$ 9XCite and 2.4-GHz 24XStream (MaxStream 2004, MaxStream 2005). This dual transceiver support allows the wireless sensing and actuation unit to operate in different regions around the world. Wireless communication poses four major constraints to the information flow within a structural monitoring and control network: bandwidth, latency, reliability, and range. It is thus important to assess the communication constraints of the transceivers.

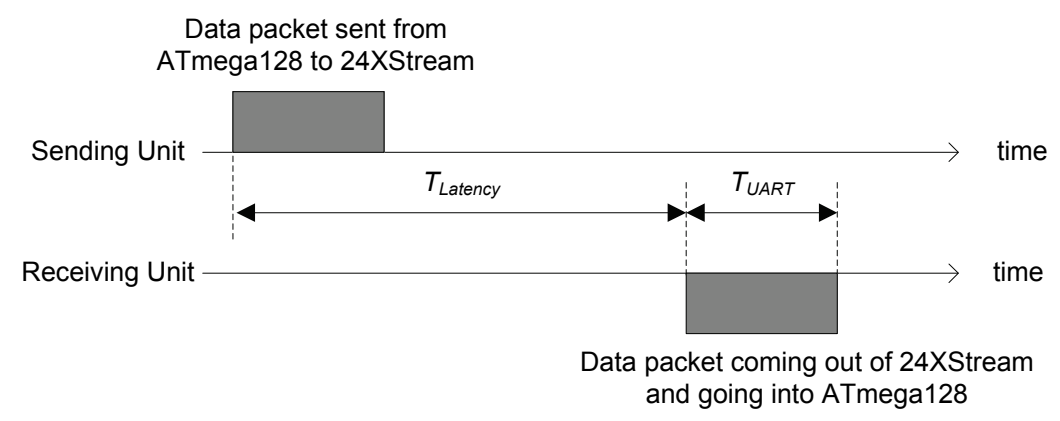

Fig. 3. Three-layer software architecture for the ATmega128 microcontroller in the wireless sensing and control unit

Bandwidth and latency are about the timing characteristics of the communication links. Bandwidth refers to the data transfer rate once a communication link is established. Using the MaxStream 24XStream transceiver as an example, the anticipated transmission time for a single data packet is illustrated in Fig. 3. The transmission time consists of the communication latency, $T_{\text {Latency }}$, of the transceivers and the time to transfer data between the microcontroller and the transceiver using the universal asynchronous receiver and transmitter (UART) interface, $T_{\text {UART }}$. Assume that the data packet to be transmitted contains $N$ bytes and the UART data rate is TUART bps (bits per second), which is equivalent to $R_{\text {UART }} / 10$ bytes per second, or $R_{U A R T} / 10000$ bytes per millisecond. It should be noted that the UART is set to transmit 10 bits for every one byte ( 8 bits) of sensor data, including one start bit and one stop bit. The communication latency in a single transmission of this data packet can be estimated as:

$$
T_{\text {SingleTransm }}=T_{\text {Latency }}+\frac{10000 \mathrm{~N}}{R_{\text {UART }}}(\mathrm{ms})
$$

In the prototype wireless sensing and control system, the setup parameters of the 24XStream transceiver are first tuned to minimize the transmission latency, $T_{\text {Latency }}$. Then experiments are conducted to measure the actual achieved $T_{\text {Latency, }}$ which turns out to be around $15 \pm 0.5 \mathrm{~ms}$. The UART data rate of the $24 \mathrm{XStream}$ radio, $R_{U A R T}$, is selected as $38400 \mathrm{bps}$ in the implementation. For example, if a data packet sent from a sensing unit to a control unit contains 11 bytes, the total time delay for a single transmission is estimated to be:

$$
T_{\text {SingleTransm }}=15+\frac{10000 \times 11}{38400} \approx 17.86(\mathrm{~ms})
$$


This amount of latency typically has minimal effect in most monitoring applications, but has noticeable effects to the timing-critical feedback control applications. This singletransmission delay represents one communication constraint that needs to be considered when calculating the upper bound for the maximum sampling rate of the control system. A few milliseconds of safety cushion time at each sampling step are a prudent addition that allows a certain amount of randomness in the wireless transmission latency without undermining the reliability of the communication system. Although the achievable transmission latency, $T_{\text {Latency, }}$ is around $15 \mathrm{~ms}$ for the MaxStream 24XStream transceiver, it can be as low as $5 \mathrm{~ms}$ for the 9XCite transceiver. This lower latency makes the 9XCite transceiver more suitable for real-time feedback control applications compared with the 24XStream transceiver. However, the 9XCite transceiver may only be used in countries and regions where the $900 \mathrm{MHz}$ band is for free public usage, such as the North America, Israel, South Korea, among others. On the other hand, operating in the $2.4 \mathrm{GHz}$ international ISM (Industrial, Science, and Medical) band, the 24XStream transceiver can be used in most countries in the world.

The other two constraints, reliability and range, are related to the attenuation of the wireless signal traveling along the transmission path. The path loss PL (in decibel) of a wireless signal is measured as the ratio between the transmitted power, $P_{T X}[\mathrm{~mW}]$, and the received power, $P_{R X}[\mathrm{~mW}]$ (Molisch 2005):

$$
P L[\mathrm{~dB}]=10 \log _{10} \frac{P_{T X}[\mathrm{~mW}]}{P_{R X}[\mathrm{~mW}]}
$$

Path loss generally increases with the distance, $d$, between the transmitter and the receiver. However, the loss of signal strength varies with the environment along the transmission path and is difficult to quantify precisely. Experiments have shown that a simple empirical model may serve as a good estimate to the mean path loss (Rappaport and Sandhu 1994):

$$
\overline{P L}(d)[\mathrm{dB}]=P L\left(d_{0}\right)[\mathrm{dB}]+10 n \log _{10}\left(d / d_{0}\right)+X_{\sigma}[\mathrm{dB}]
$$

Here $P L\left(d_{0}\right)$ is the free-space path loss at a reference point close to the signal source $\left(d_{0}\right.$ is usually selected as approximately 1 meter). $X_{\sigma}$ represents the variance of the path loss, which is a zero-mean log-normally-distributed random variable with a standard deviation of $\sigma$. The parameter $n$ is the path loss exponent that describes how fast the wireless signal attenuates over distance. Basically, Eq. (4) indicates an exponential decay of signal power:

$$
P_{R X}[\mathrm{~mW}]=P_{0}[\mathrm{~mW}]\left(\mathrm{d} / d_{0}\right)^{-n}
$$

where $P_{0}$ is the received power at the reference distance $d_{0}$. Typical values of $n$ are reported to be between 2 and 6 . Table 2 shows examples of measured $n$ and $\sigma$ values in different buildings for $914 \mathrm{MHz}$ signals (Rappaport and Sandhu 1994).

A link budget analysis can be used to estimate the range of wireless communication (Molisch 2005). To achieve a reliable communication link, it is required that

$$
P_{T X}[\mathrm{dBm}]+A G[\mathrm{dBi}] \geq P L(d)[\mathrm{dB}]+R S[\mathrm{dBm}]+F M[\mathrm{~dB}]
$$


where $A G$ denotes the total antenna gain for the transmitter and the receiver, $R S$ the receiver sensitivity, FM the fading margin to ensure quality of service, and $P L(d)$ the realized path loss at some distance $d$ within an operating environment. Table 3 summarizes the link budget analysis for the 9XCite and 24XStream transceivers, and their estimated indoor ranges.

\begin{tabular}{lcc}
\hline Building & $n$ & $\sigma[\mathrm{dB}]$ \\
\hline Grocery store & 1.8 & 5.2 \\
Retail store & 2.2 & 8.7 \\
Suburban office building - open plan & 2.4 & 9.6 \\
Suburban office building - soft partitioned & 2.8 & 14.2 \\
\hline
\end{tabular}

Table 2. Values of path loss exponent $n$ at $914 \mathrm{MHz}$

\begin{tabular}{lcc}
\hline & 9XCite & 24XStream \\
\hline$P_{T X}[\mathrm{dBm}]$ & 0.00 & 16.99 \\
$A G[\mathrm{dBi}]$ & 4.00 & 4.00 \\
$R S[\mathrm{dBm}]$ & -104.00 & -105.00 \\
$F M[\mathrm{~dB}]$ & 22.00 & 22.00 \\
$\overline{P L}=P_{T X}+A G-R S-F M[\mathrm{~dB}]$ & 86.00 & 103.99 \\
$P L\left(d_{0}\right)[\mathrm{dB}], d_{0}=1 \mathrm{~m}$ & 31.53 & 40.05 \\
$\overline{P L}-P L\left(d_{0}\right)[\mathrm{dB}]$ & 54.47 & 63.94 \\
$n$ & 2.80 & 2.80 \\
$\bar{d}[\mathrm{~m}]$ & 88.20 & 192.18 \\
\hline
\end{tabular}

Table 3. Link budget analysis to the wireless transceivers

The path loss exponent $n$ is selected to be 2.8 , which is the same as the soft-partitioned office building in Table 2. Generally, $2.4 \mathrm{GHz}$ signals typically have higher attenuation than $900 \mathrm{MHz}$ signals, and, thus, a larger path loss exponent $n$. The transmitter power $P_{T X}$, receiver sensitivity $R S$, and fading margin $F M$ of the two wireless transceivers are obtained from the MaxStream datasheets. A total antenna gain AG of 4 is employed by assuming that low-cost $2 \mathrm{dBi}$ whip antennas are used by both the transmitting and the receiving sides. The free-space path loss at $d_{0}$ is computed using the Friis transmission equation (Molisch 2005):

$$
P L\left(d_{0}\right)[\mathrm{dB}]=20 \log _{10}\left(4 \pi d_{0} / \lambda\right)
$$

where $\lambda$ is the wavelength of the corresponding wireless signal. Finally, assuming that the variance $X_{\sigma}$ is zero, the mean communication range $\bar{d}$ can be derived from Eq. (4) as:

$$
\bar{d}=d_{0} 10^{\left(\overline{P L}-P L\left(d_{0}\right)\right) /(10 n)}
$$

Table 3 shows that the transceivers can achieve the communication ranges indicated in Table 1. It is important to note the sensitivity of the communication range with respect to the path loss exponent $n$ in Eq. (8). For instance, if the exponent of 3.3 for indoor traveling (through brick walls, as reported by Janssen \& Prasad (1992) for $2.4 \mathrm{GHz}$ signals) is used for the 24XStream transceiver, its mean communication range reduces by half to $87 \mathrm{~m}$. 


\section{Wireless structural health monitoring}

The prototype wireless unit is first investigated for applications in wireless structural health monitoring. A structural health monitoring system measures structural performance and operating conditions with various types of sensing devices, and evaluates structural safety using damage diagnosis or prognosis methods. Eliminating lengthy cables, wireless sensor networks can offer a low-cost alternative to traditional cable-based structural health monitoring systems. Another advantage of a wireless system is the ease of relocating sensors, thus providing a flexible and easily reconfigurable system architecture. This section first provides an overview to the wireless structural health monitoring system, and then introduces the communication protocol design for reliable data management in the prototype system. A large-scale field deployment of the wireless structural health monitoring system is summarized at the end of the section.

\subsection{Overview of the wireless structural health monitoring system}

A simple star-topology network is adopted for the prototype wireless sensing system. The system includes a server and multiple structural sensors, signal conditioning modules, and wireless sensing units (Fig. 4). The server is used to organize and collect data from multiple wireless sensing units in the sensor network. The server is responsible for: 1) commanding all the corresponding wireless sensing units to perform data collection or interrogation tasks, 2) synchronizing the internal clocks of the wireless sensing units, 3) receiving data or analysis results from the wireless network, and 4) storing the data or results. Any desktop or laptop computer connected with a compatible wireless transceiver can be used as the server. The server can also provide Internet connectivity so that sensor data or analysis results can be viewed remotely from other computers over the Internet. Since the server and the wireless sensing units must communicate frequently with each other, portions of their software are designed in tandem to allow seamless integration and coordination.

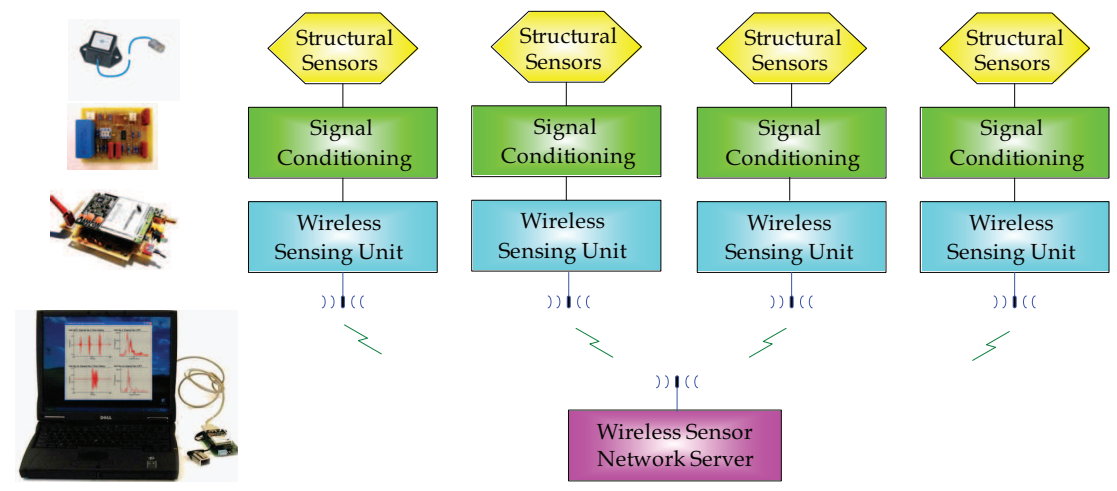

Fig. 4. An overview of the prototype wireless structural sensing system

At the beginning of each wireless structural sensing operation, the server issues commands to all the units, informing the units to restart and synchronize. After the server confirms that all the wireless sensing units have restarted successfully, the server queries the units one by one for the data they have thus far collected. Before the wireless sensing unit is queried for its data, the data is temporarily stored in the unit's onboard SRAM memory buffer. 
A unique feature of the embedded wireless sensing unit software is that it can continue collecting data from interfaced sensors in real-time as the wireless sensing unit is transmitting data to the server. In its current implementation, at each instant in time, the server can only communicate with one wireless sensing unit. In order to achieve real-time continuous data collection from multiple wireless sensing units with each unit having up to four analog sensors attached, a dual stack approach has been implemented to manage the SRAM memory (Wang, et al. 2007a). When a wireless sensing unit starts collecting data, the embedded software establishes two memory stacks dedicated to each sensing channel for storing the sensor data. For each sensing channel, at any point in time, only one of the stacks is used to store the incoming data stream. While incoming data is being stored into the dedicated memory stack, the system transfers the data in the other stack out to the server. For each sensing channel, the role of the two memory stacks alternate as soon as one stack is filled with newly collected data.

\subsection{Communication design of the wireless structural health monitoring system}

To ensure reliable wireless communication between the server and the wireless units, the communication protocol needs to be carefully designed and implemented. The commonly used network communication protocol is the Transmission Control Protocol (TCP) standard. TCP is a sliding window protocol that handles both timeouts and retransmissions. It establishes a full duplex virtual connection between two endpoints. Although TCP is a reliable communication protocol, it is too general and cumbersome to be employed by the low-power and low data-rate communication such as in a wireless structural sensing network. The relatively long latency of transmitting each wireless packet is another bottleneck that may slow down the communication throughput. For practical and efficient application in a wireless structural sensing network, a simpler communication protocol is needed to minimize transmission overhead. Yet the protocol has to be designed to ensure reliable wireless transmission by properly addressing possible data loss. The communication protocol designed for the prototype wireless sensing system inherits some useful features of TCP, such as data packetizing, sequence numbering, timeout checking, and retransmission. Based upon pre-assigned arrangement between the server and the wireless units, the sensor data stream is segmented into a number of packets, each containing a few hundred bytes. A sequence number is assigned to each packet so that the server can request the data sequentially.

To simplify the communication protocol, special characteristics of the structural health monitoring application are exploited. For example, since the objective in structural monitoring application is normally to transmit sensor data or analysis results to the server, the server is assigned the responsibility for ensuring reliable wireless communication. As the server program normally runs on a computer and the wireless unit program runs on a microcontroller, it is also reasonable to assign the responsibility to the server since it has much higher computing power. For example, communication is always initiated by the server. After the server sends a command to the wireless sensing unit, if the server does not receive an expected response from the unit within a certain time limit, the server will resend the last command again until the expected response is received. However, after a wireless sensing unit sends a message to the server, the unit does not check if the message has arrived at the server correctly or not, because the communication reliability is assigned to the server. The wireless sensing unit only becomes aware of the lost data when the server queries the unit for the same data again. In other words, the server plays an "active" role in the communication protocol while the wireless sensing unit plays more of a "passive" role. 


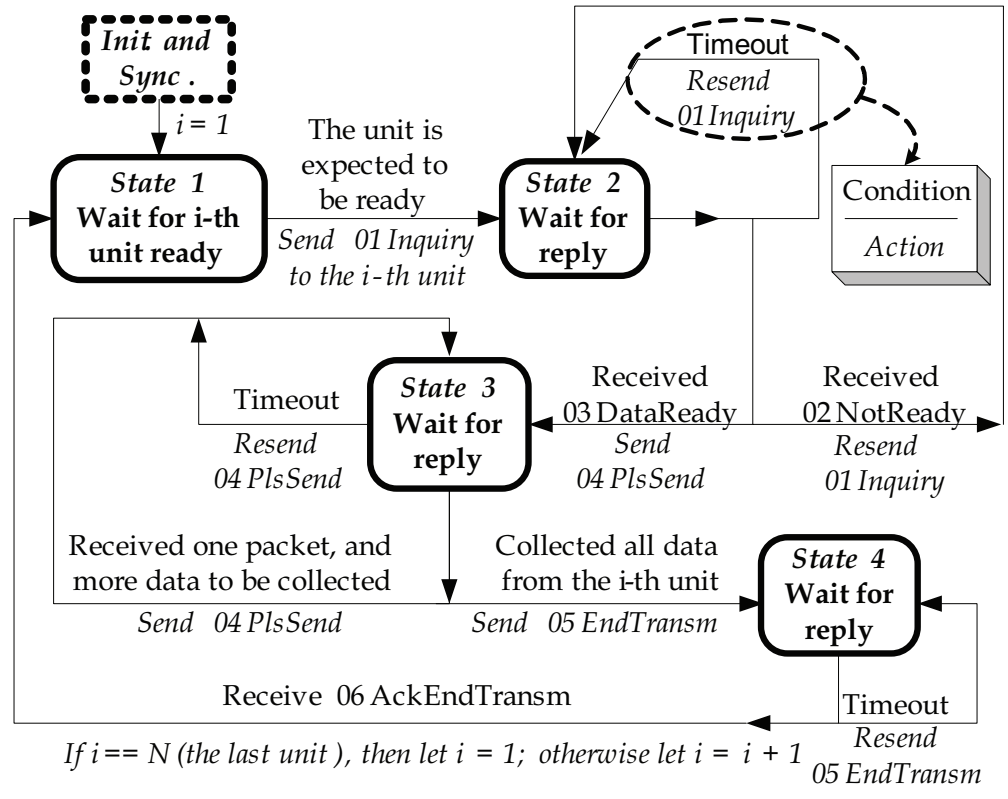

(a) State diagram of the server

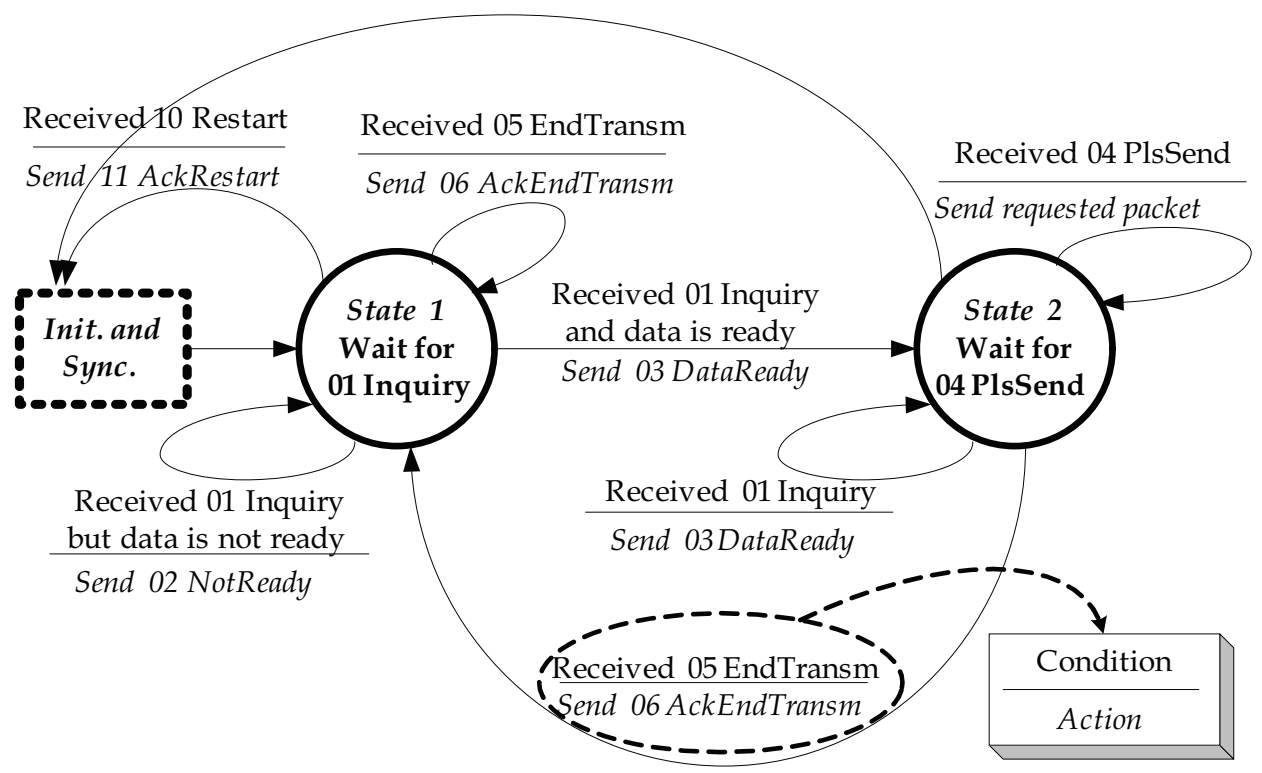

(b) State diagram of a wireless sensing unit.

Fig. 5. Communication state diagrams for wireless structural health monitoring 
Finite state machine concepts are employed in designing the communication protocol for the wireless sensing units and the server. A finite state machine consists of a set of states and definable transitions between the states (Tweed 1994). At any point in time, the state machine can only be in one of the possible states. In response to different events, the state machine transits between its discrete states. The communication protocol for initialization and synchronization can be found in (Wang, et al. 2007a). Fig. 5(a) shows the communication state diagram of the server for one round of sensor data collection, and Fig. 5(b) shows the corresponding state diagram of the wireless units. During each round of data collection, the server collects sensor data from all of the wireless units; note that the server and the units have separate sets of state definitions.

At the beginning of data collection, the server and all the units are all set in State 1. Starting with the first wireless unit in the network, the server queries the sensor for the availability of data by sending the '01Inquiry' command. If the data is not ready, the unit replies '02NotReady', otherwise the unit replies '03DataReady' and transits to State 2. After the server ensures that the data from this wireless unit is ready for collection, the server transits to State 3. To request a data segment from a unit, the server sends a '04PlsSend' command that contains a packet sequence number. One round of data collection from one wireless unit is ended with a two-way handshake, where the server and the unit exchange '05EndTransm' and '06AckEndTransm' commands. The server then moves on to the next unit and continuously collects sensor data round-by-round.

\subsection{Field validation tests at Voigt Bridge}

Laboratory and field validation tests have been conducted to verify the performance of the wireless structural monitoring system. Field tests are particularly helpful in assessing the limitations of the system, and providing valuable experience that can lead to further improvements in the system hardware and software design. This section presents an overview of the validation tests conducted on the Voigt Bridge located on the campus of the University of California, San Diego (UCSD) in La Jolla, California (Fraser, et al. 2006). Voigt Bridge is a two lane concrete box girder highway bridge. The bridge is about $89.4 \mathrm{~m}$ long and consists of four spans (Fig. 6). The bridge deck has a skew angle of $32^{\circ}$, with the concrete box-girder supported by three single-column bents. Over each bent, a lateral diaphragm with a thickness of about $1.8 \mathrm{~m}$ stiffens the girder. Longitudinally, the box girder is partitioned into five cells running the length of the bridge (Fig. 6b).

Girder cells along the north side of the bridge are accessible through four manholes on the bridge sidewalk. As a testbed project for structural health monitoring research, a cablebased system has been installed in the northern-most cells of the box girder. The cable-based system includes accelerometers, strain gages, thermocouples, and humidity sensors. For the purpose of validating the proposed wireless structural monitoring system, thirteen accelerometers interfaced to wireless sensing units are installed within the two middle spans of the bridge to measure vertical vibrations. One wireless sensing unit (associated with one signal conditioning module and one accelerometer) is placed immediately below the accelerometer associated with the permanent wired monitoring system. While the wired accelerometers are mounted to the cell walls, wireless accelerometers are simply mounted on the floor of the girder cells to expedite the installation process. The installation and calibration of the wireless monitoring system, including the placement of the 13 wireless sensors, takes about an hour. The MaxStream 9XCite wireless transceiver operating at $900 \mathrm{MHz}$ is integrated with each wireless sensing unit. 


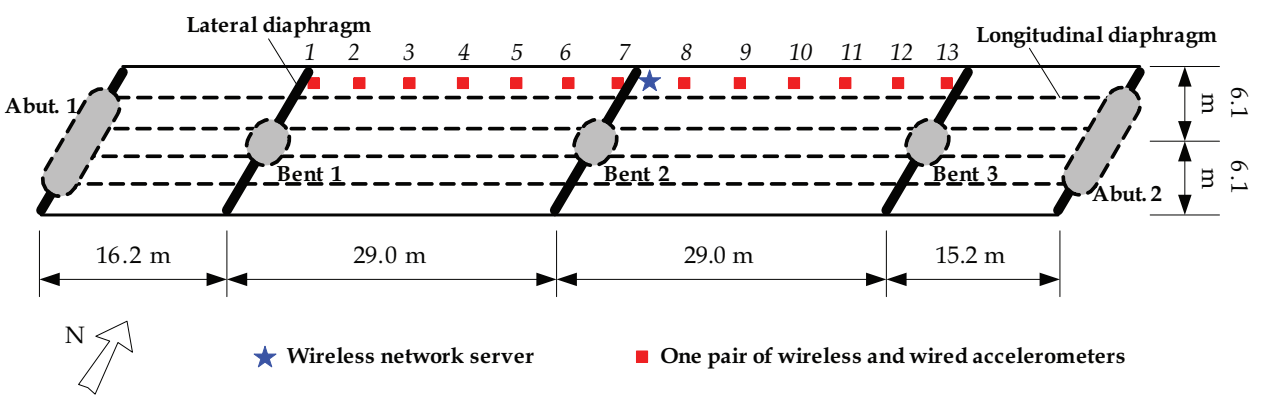

(a) Plan view of the bridge illustrating locations of wired and wireless sensing systems

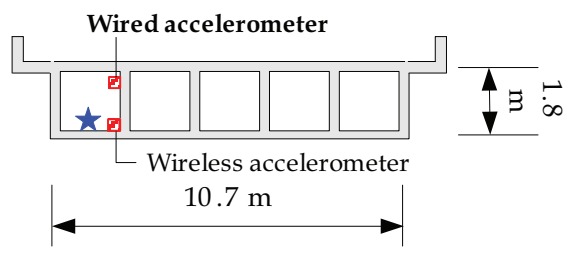

$\underline{\text { Section A- A }}$

(b) Elevation view to section A-A

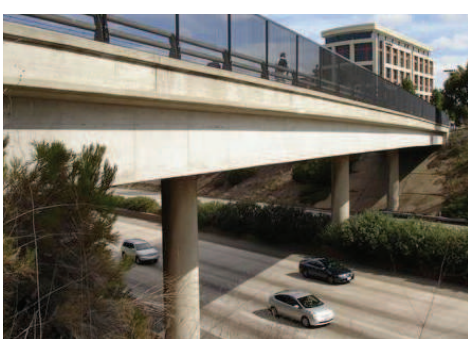

(c) Side view of the bridge over Interstate 5

Fig. 6. Voigt Bridge test comparing the wireless and wired sensing systems

Two types of accelerometers are associated with each monitoring system. At locations \#3, 4, 5, 9, 10, and 11 in Fig. 6(a), PCB Piezotronics 3801 accelerometers are used with both the cabled and the wireless systems. At the other seven locations, Crossbow CXL01LF1 accelerometers are used with the cabled system, while Crossbow CXL02LF1Z accelerometers are used with the wireless system. Table 4 summarizes the key parameters of the three types of accelerometers. Signal conditioning modules are used for filtering noise, amplifying and shifting signals for the wireless accelerometers. The signals of the wired accelerometers are directly digitized by a National Instruments PXI-6031E data acquisition board (Fraser, et al. 2006). Sampling frequencies for the cable-based system and the wireless system are 1,000 Hz and $200 \mathrm{~Hz}$, respectively.

\begin{tabular}{cccc}
\hline Specification & PCB3801 & CXL01LF1 & CXL02LF1Z \\
\hline Sensor Type & Capacitive & Capacitive & Capacitive \\
Maximum Range & $\pm 3 \mathrm{~g}$ & $\pm 1 \mathrm{~g}$ & $\pm 2 \mathrm{~g}$ \\
Sensitivity & $0.7 \mathrm{~V} / \mathrm{g}$ & $2 \mathrm{~V} / \mathrm{g}$ & $1 \mathrm{~V} / \mathrm{g}$ \\
Bandwidth & $80 \mathrm{~Hz}$ & $50 \mathrm{~Hz}$ & $50 \mathrm{~Hz}$ \\
RMS Resolution (Noise Floor) & $0.5 \mathrm{mg}$ & $0.5 \mathrm{mg}$ & $1 \mathrm{mg}$ \\
Minimal Excitation Voltage & $5 \sim 30 \mathrm{VDC}$ & $5 \mathrm{VDC}$ & $5 \mathrm{VDC}$ \\
\hline
\end{tabular}

Table 4. Parameters of the accelerometers used by the wire-based and wireless systems in the Voigt Bridge test 

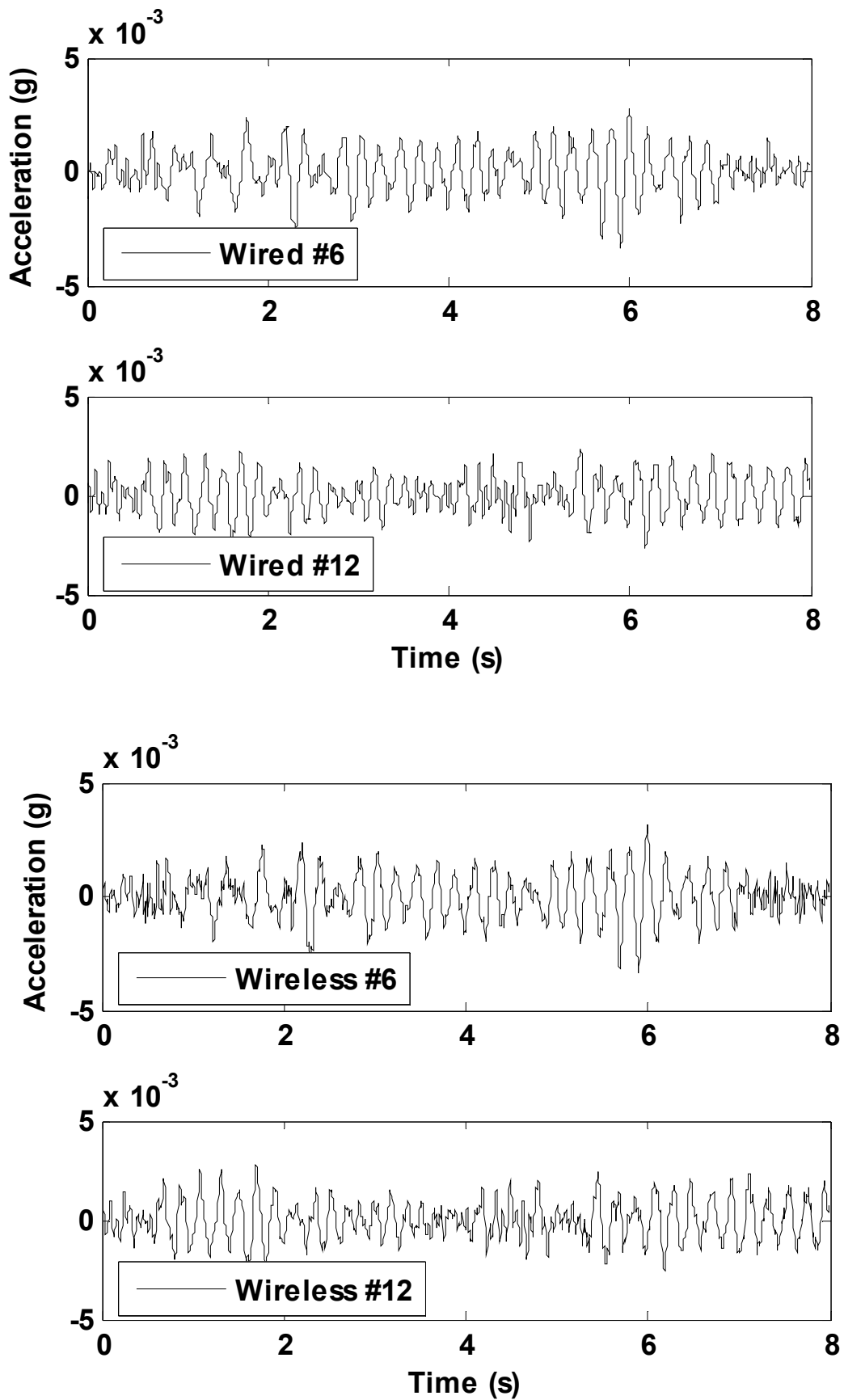

(a) Comparison between wired and wireless time history data 

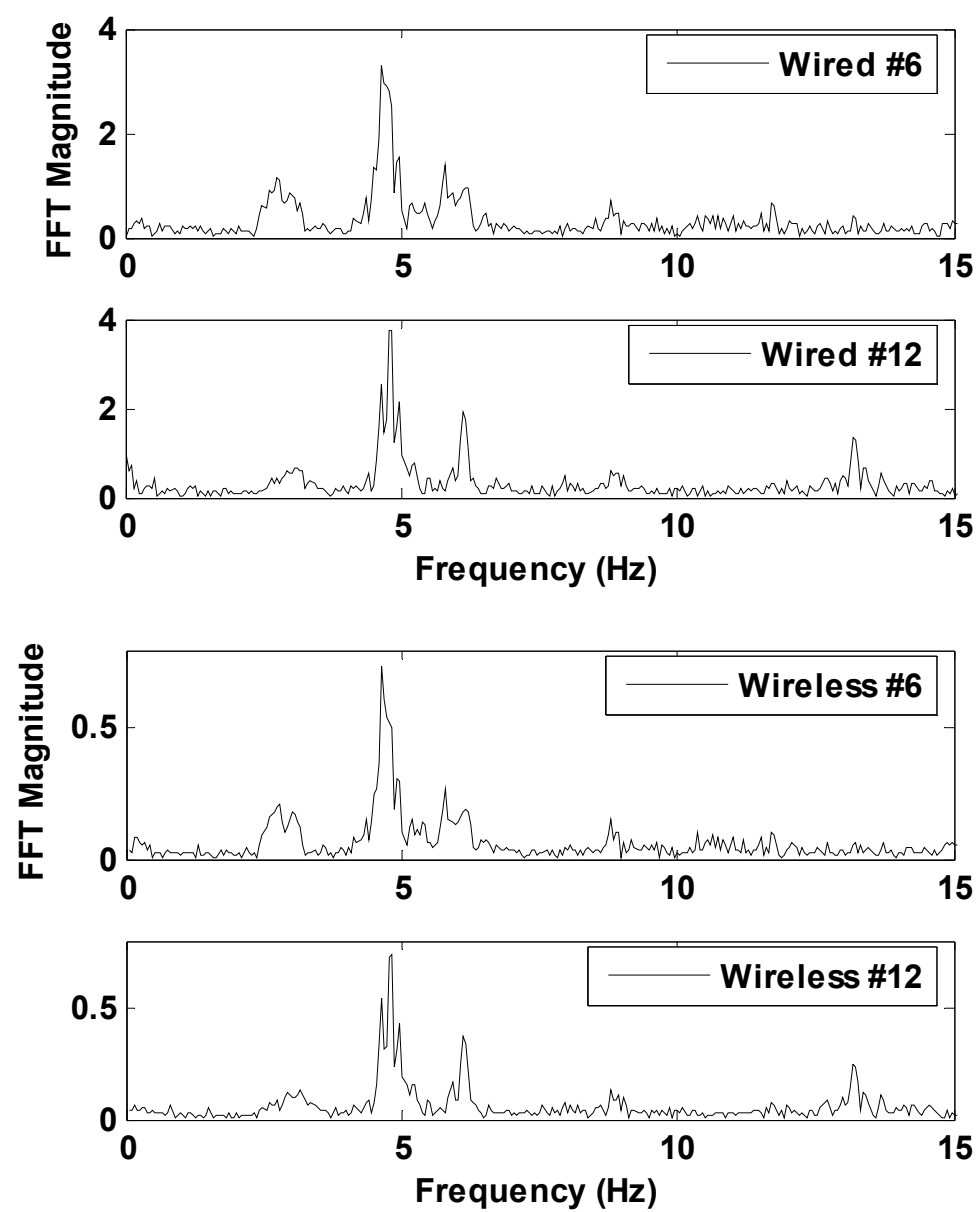

(b) Comparison between FFT to the wired data, as computed offline by a computer, and FFT to the wireless data, as computed online by the wireless sensing units

Fig. 7. Comparison between wired and wireless data for the Voigt Bridge test

The bridge is under normal traffic operation during the tests. Fig. 7(a) shows the time history data at locations \#6 and \#12, collected by the cable-based and wireless monitoring systems when a vehicle passes over the bridge. A close match is observed between the data collected by the two systems. The minor difference between the two data sets can be mainly attributed to two sources: 1) the signal conditioning modules are used in the wireless system but not in the cabled system; 2) the wired and wireless accelerometer locations are not exactly adjacent to each other, as previously described. Fig. 7(b) shows the Fourier spectra determined from the time history data. The FFT results using the data collected by the cabled system are computed offline, while the FFT results corresponding to the wireless data are computed online in real-time by each wireless sensing unit. After each wireless sensing unit executes its FFT algorithm, the FFT results are wirelessly transmitted to the 
network server. Strong agreement between the two sets of FFT results validates the computational accuracy of the wireless sensing units. It should be pointed out that because the sampling frequency of the cabled system is five times higher than that of the wireless system, the magnitude of the Fourier spectrum for the wired data is also about five times higher than those for the wireless data.

One attractive feature of the wireless sensing system is that the locations of the sensors can be re-configured easily. To determine the operating deflection shapes of the bridge deck, the configuration of the original wireless sensing system is changed to attain a more suitable spatial distribution. Twenty wireless accelerometers and the wireless network server are mounted to the bridge sidewalks (Fig. 8). The communication distance between the server and the farthest wireless sensing unit is close to the full length of the bridge. The installation and calibration of the wireless monitoring system, including the placement of all the wireless sensors, again takes about an hour. Sampling frequency for the wireless monitoring system is kept at $200 \mathrm{~Hz}$.

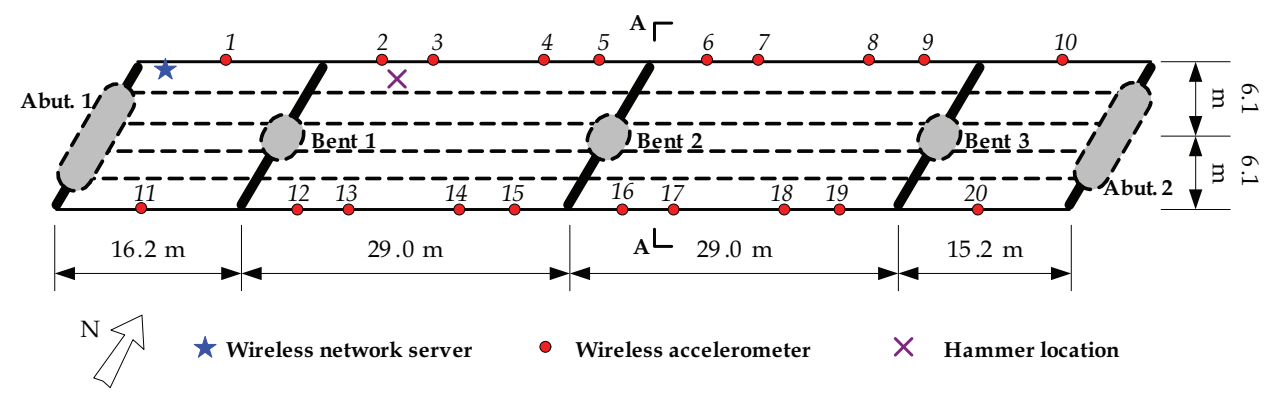

(a) Plan view of the bridge illustrating locations of wireless accelerometers

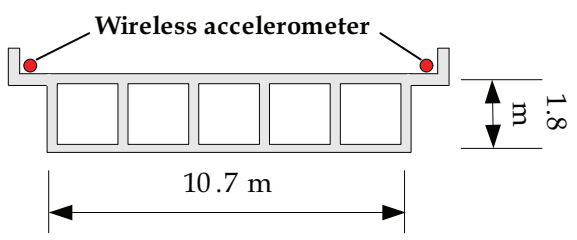

$\underline{\text { Section A-A }}$

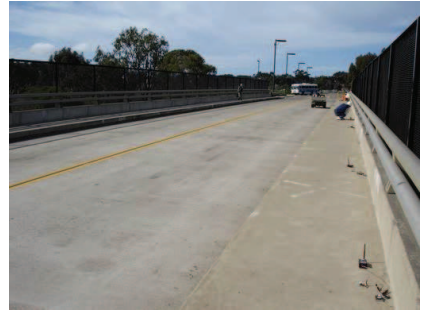

(c) Side view of the bridge over Interstate 5

(b) Elevation view to section A-A

Fig. 8. Wireless accelerometer deployment for the operating deflection shape analysis to Voigt Bridge

The communication protocol described before is implemented in the server and the wireless sensing units. For the tests described in this chapter, the server collects sensor data or FFT results from all 20 wireless units. Due to the length of the bridge and continuous traffic conditions, the wireless communication experienced some intermittent difficulty during the two days of field testing. However, the wireless monitoring system proved robust by recognizing communication failures and successfully retransmitting the lost data according to the communication protocol rules. 
Fig. 9 shows the operating deflection shapes (ODS) extracted from one set of test data collected during a hammer excitation test. The hammer excitation is applied at the location shown in Fig. 8(a) and during intervals of no passing vehicles. DIAMOND, a modal analysis software package, is used to extract the operating deflection shapes (ODS) of the bridge deck (Doebling, et al. 1997). Under hammer excitation, the operating deflection shapes at or near a resonant frequency should be dominated by a single mode shape (Richardson 1997). Fig. 9 presents the first four dominant operating deflection shapes of the bridge deck using wireless acceleration data. The ODS \#1 $(4.89 \mathrm{~Hz})$, \#2 (6.23 Hz), and \#4 (11.64 Hz) show primarily flexural bending modes of the bridge deck; a torsional mode is observed in ODS \#3 $(8.01 \mathrm{~Hz})$. Successful extraction of the ODS shows that the acceleration data from the 20 wireless units are well synchronized.
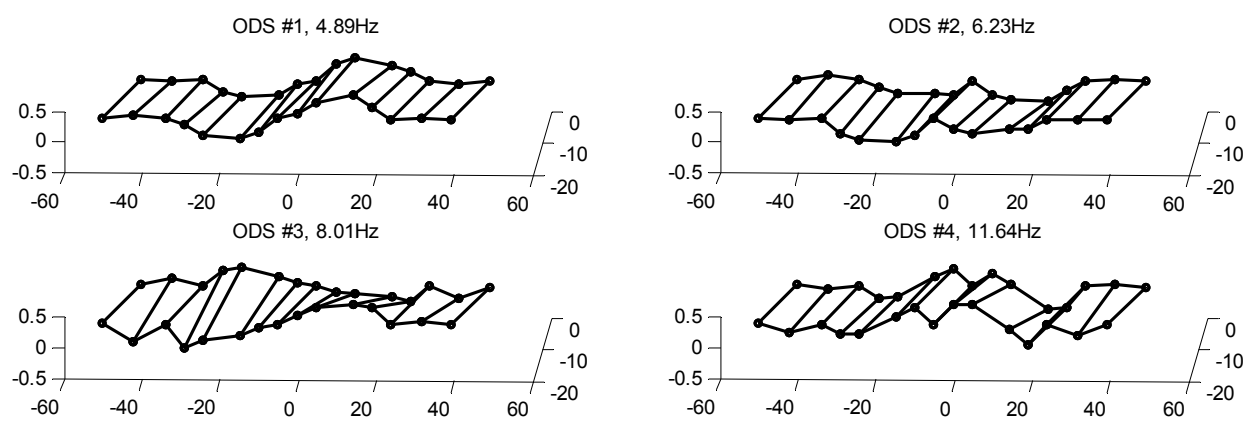

Fig. 9. Operating deflection shapes extracted from wireless sensor data

\section{Wireless structural control}

A feedback structural control system contains an integrated network of sensors, controller, and control devices. When external excitation (such as an earthquake or typhoon) occurs, structural response is measured by sensors and immediately collected by the controller. The controller makes optimal decisions for the control devices, which then exert appropriate forces to the structure so that undesired structural vibrations are effectively mitigated. A wireless sensing/control unit can serve as both the sensor and the controller modules of a structural control system. Each wireless unit, in addition to collecting and communicating sensor data in real time, can also make optimal control decisions and command control devices. This section first provides an overview to the prototype wireless structural control system, and then describes the communication protocol design of the system. Laboratory wireless structural control experiments are also reported.

\subsection{Overview of the wireless structural control system}

Fig. 10 illustrates the communication patterns of a centralized control system using cabled communication and the prototype decentralized structural control system using wireless communication. In a centralized structural control system, one centralized controller collects data from all the sensors in the whole structure, computes control decisions, and then dispatches command signals to control devices. This centralized control strategy implemented with cabled communication requires high instrumentation cost, is difficult to reconfigure, 
and potentially suffers from single-point failure at the controller. Wireless decentralized control architectures can offer an alternative solution. In a decentralized architecture, multiple sensors and controllers can be distributively placed in a large structure, where the controller nodes can be closely collocated with the control devices. As each controller only needs to communicate with sensors and control devices in its vicinity, the requirement on communication range can be significantly reduced, and the communication latency decreases by reducing the number of sensors or control devices that each controller has to communicate with.
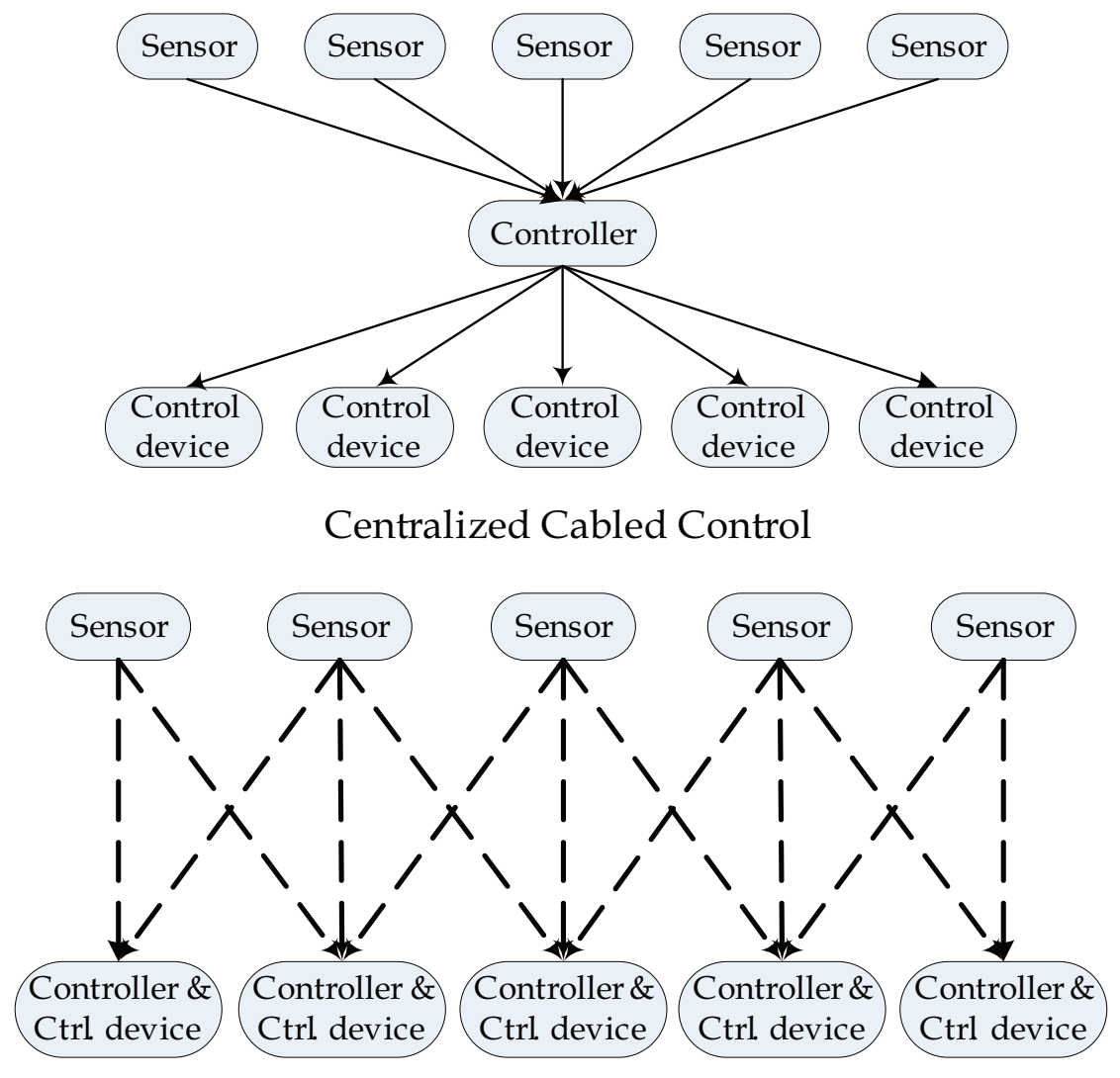

\section{Decentralized Wireless Control}

Fig. 10. Centralized and decentralized control systems

For application in wireless feedback structural control, real-time communication is important for system performance. Limited wireless communication range poses another challenge while instrumenting a large-scale structure with the wireless sensing and control system. Particularly, in discrete-time feedback control, a steady sampling time step and low communication latency are essential for the system performance. The feedback control loop designed for the prototype wireless sensing and control system is 
illustrated in Fig. 11(a), and the pseudo code implementing the feedback loop is presented in Fig. 11(b). As shown in the figures, sensing is designed to be clock-driven, while control is designed to be event-driven. The wireless sensing nodes collect sensor data at a preset sampling rate, and transmit the data during an assigned time slot. Upon receiving the required sensor data, the control nodes immediately compute control decisions and apply the corresponding command signals to the control devices. If due to occasional data packet loss, a control node doesn't receive the expected sensor data at one time step, the control node may use a projected data sample for control decisions, or doesn't take any action at this time step.

\subsection{Communication protocol design for the wireless structural control system}

Similar to the structural monitoring application, a reliable communication protocol must be properly designed for the wireless structural control system. Fig. 12 illustrates the communication state diagrams of a coordinator unit and other wireless units within a wireless sensing and control subnet. To initiate the system operation, the coordinator unit first broadcasts a start command '01StartCtrl' to all other sensing and control units. Once the start command and its acknowledgement '03AcknStartCtrl' are received, the system starts real-time feedback control operation, i.e. both the coordinator and other units are in State 2.

\section{Wireless Sensor Nodes Wireless Control Nodes}

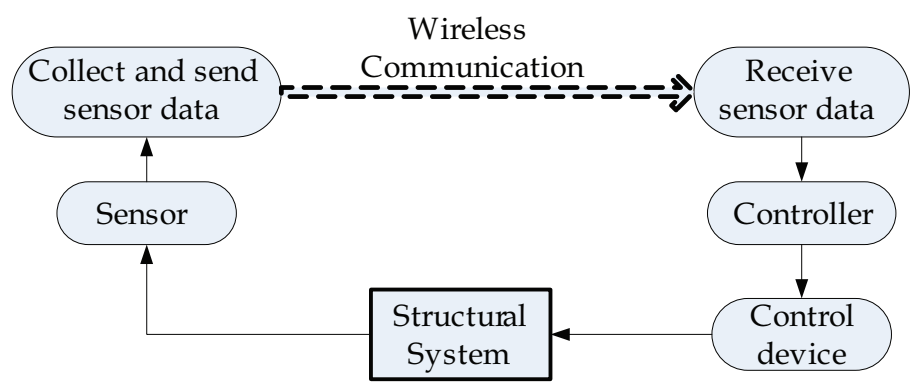

(a) Feedback control loop between the wireless sensing nodes and control nodes

\begin{tabular}{|c|c|}
\hline $\begin{array}{l}\text { Wireless Sensing Nodes } \\
\text { (Clock-driven) }\end{array}$ & $\begin{array}{l}\text { Wireless Control Nodes } \\
\text { (Event-driven) }\end{array}$ \\
\hline ITERATE \{ & ITERATE \{ \\
\hline & IF (sensor data arrived on time) \\
\hline Wait for the assigned time slot. & Compute control decisions. \\
\hline & Apply control command signal. \\
\hline Sample sensor data. & ELSE \\
\hline & Use projected data sample or no action. \\
\hline Wirelessly transmit sensor data. $\longrightarrow$ & Wait for the wireless sensor data. \\
\hline$\}$ & \} \\
\hline
\end{tabular}

(b) Pseudo code for the feedback control loop

Fig. 11. Illustration of the feedback control loop in a wireless decentralized control system 
At every sampling time step, the coordinator unit broadcasts a beacon signal '02BeaconData' together with its own sensor data, announcing the start of a new time step. Upon receiving the beacon signal, other sensing units broadcast their sensor data following a preset transmission sequence, so that transmission collision is avoided. The wireless control units responsible for commanding the control devices receive the sensor data, calculate desired control forces, and apply control commands at each time step. In order to guarantee a constant sampling time step and to minimize feedback latency, timeout checking or retransmission is not recommended during the feedback control operation. This design is suitable for both centralized control and decentralized control.

\section{Coordinator Unit}

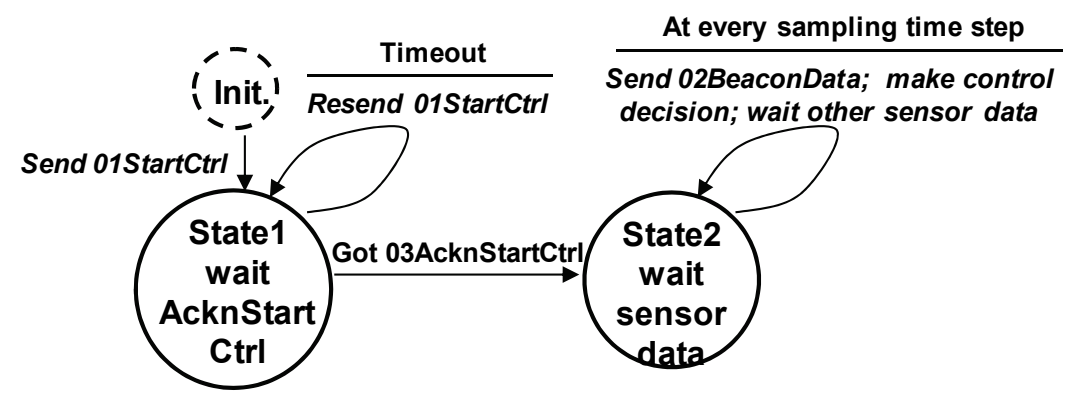

Other Sensing/Control Units

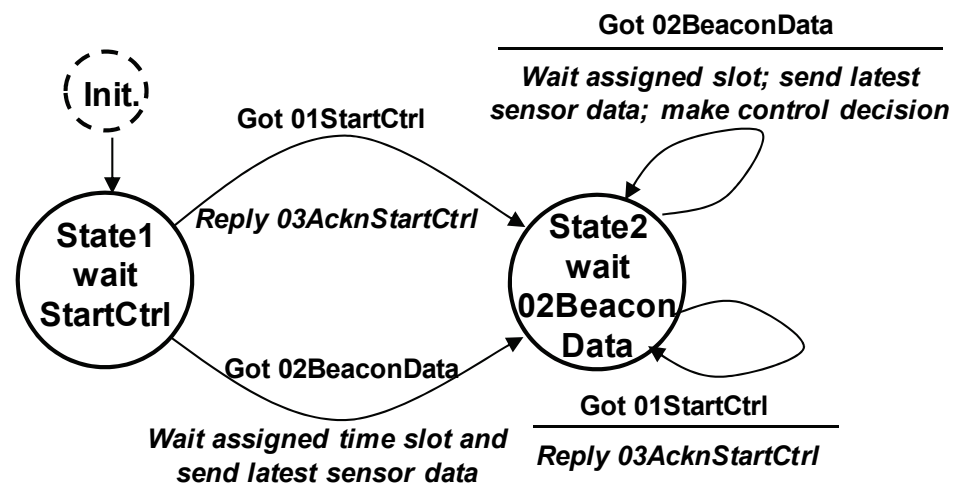

Fig. 12. Communication state diagram of a coordinator unit and other sensing/control units in one wireless subnet

For illustration purpose, a 3-story structure instrumented with the prototype wireless control system is shown in Fig. 13. The steel frame structure is designed and constructed by researchers affiliated with the National Center for Research on Earthquake Engineering (NCREE) in Taipei, Taiwan. The prototype wireless system consists of wireless sensors and controllers that are mounted on the structure for measuring structural response data and commanding MR dampers in real-time. Besides the wireless sensing and control units 
that are necessary for data collection and the operation of the control devices, a remote command server with a wireless transceiver is also included for experimental purpose. In a laboratory setup, the server is designed to initiate the operation of the control system and to log the data flow in the wireless network. To initiate the operation, the command server first broadcasts a start signal to all the wireless sensing and control units. Once the start command is received, the wireless units that are responsible for collecting sensor data start acquiring and broadcasting data at a preset time interval. Accordingly, the wireless units responsible for commanding the MR dampers receive the sensor data, calculate desired control forces, and apply control commands within the specified time interval.

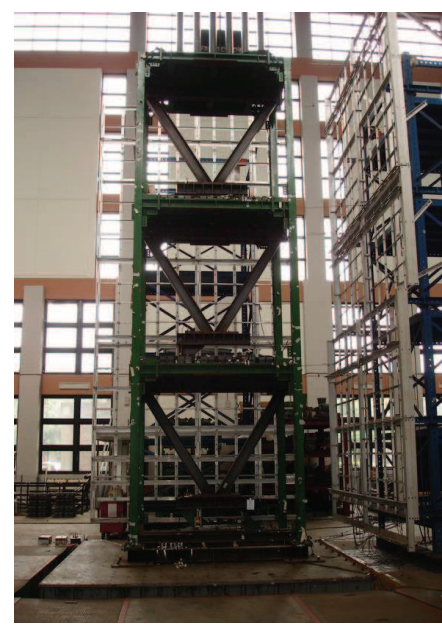

(a) A 3-story test structure mounted on the shake table

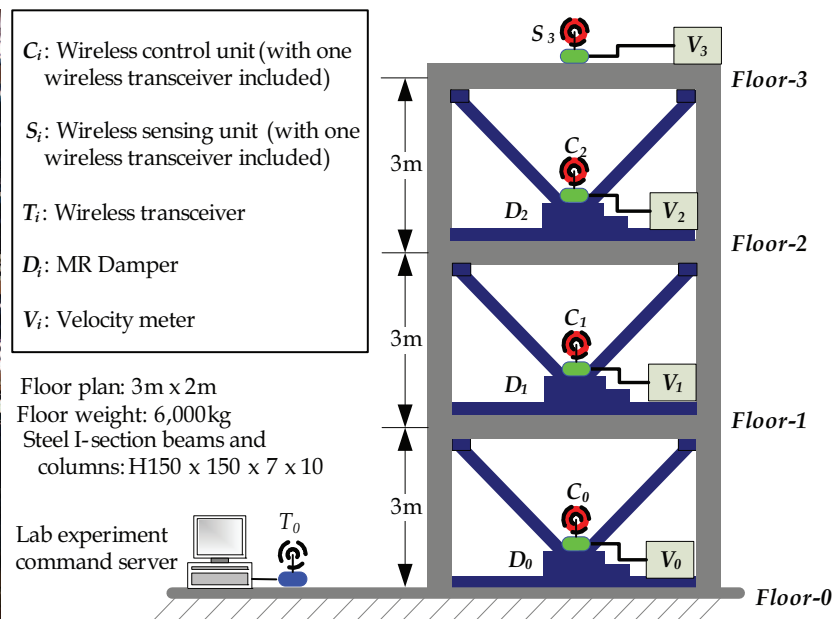

(b) Deployment of the wireless sensors, controllers, and control devices

Fig. 13. Laboratory setup of the wireless structural control system

To coordinate the wireless transmissions during the feedback control, a pre-specified communication sequence should be observed by all the wireless units. For example, if all three wireless control units need velocity data from all the floors to compute control decisions, a communication sequence illustrated in Fig. 14 can be adopted by the prototype system. The control sampling step, which is $80 \mathrm{~ms}$ in this example, is mostly decided by the total time required for transmitting all four data packets. For the 24XStream wireless transceiver adopted in the system, wireless transmission of each velocity measurement takes about $18 \mathrm{~ms}$. During every control time step, the wireless unit $C_{0}$ first samples the velocity data $V_{0}$ at its own floor, and then sends out the data together with a beacon signal to other wireless units. Upon receiving the beacon signal, units $C_{1}, C_{2}$, and $S_{3}$ sequentially broadcast their sensor data. Last, a period of $8 \mathrm{~ms}$ is designed as a safety cushion for each control sampling time step, allowing certain randomness in the wireless transmission time. The control units $C_{0}, C_{1}$, and $C_{2}$ compute control decisions and apply actuation signals during the intervals of wireless transmissions. 


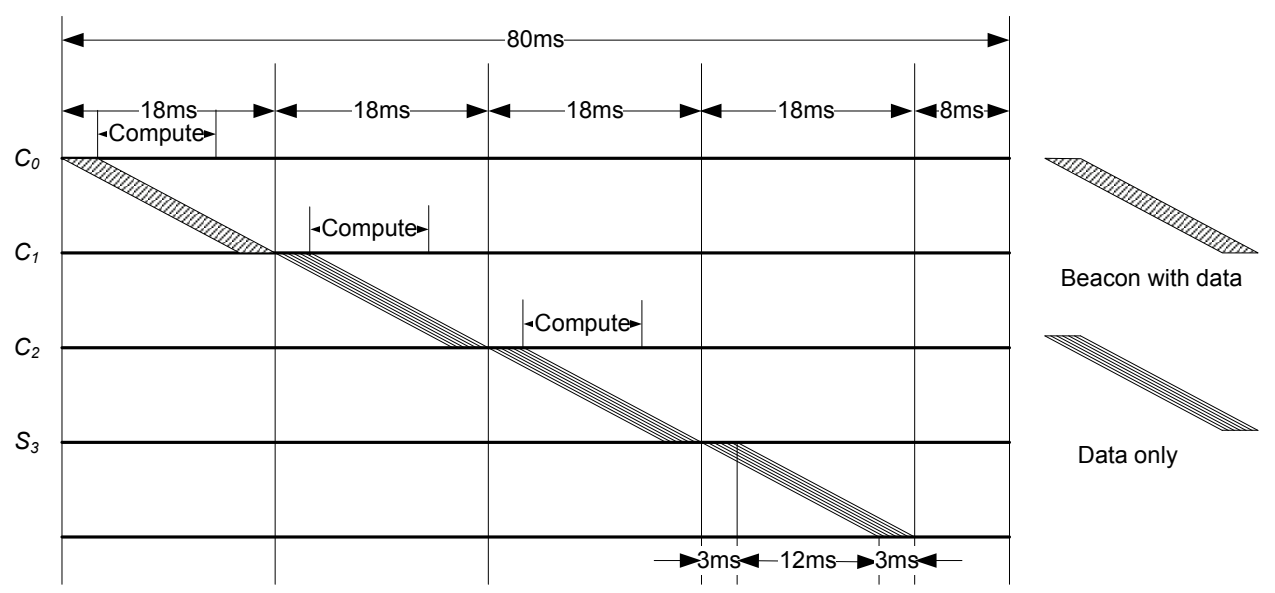

Fig. 14. Communication sequence in a wireless structural control network

\subsection{Validation experiments for the wireless structural control system}

Validation experiments for the wireless control system were conducted at NCREE in Taipei, Taiwan, using the structure shown in Fig. 13. The floor plan of this structure is $3 \mathrm{~m} \times 2 \mathrm{~m}$, with each floor weight adjusted to $6,000 \mathrm{~kg}$ using concrete blocks; inter-story heights are $3 \mathrm{~m}$. The three-story structure is mounted on a $5 \mathrm{~m} \times 5 \mathrm{~m}$ 6-DOF shake table. For this study, only longitudinal excitation in one degree of freedom is applied. Besides wireless sensors, a separate set of accelerometers, velocity meters, and linear variable displacement transducers (LVDT) are installed on each floor of the structure; this set of sensors are interfaced to a high-precision tethered data acquisition (DAQ) system native to the NCREE facility.

For this experimental study, three $20 \mathrm{kN}$ MR dampers are deployed. Each damper is installed under a V-brace upon one of the three floors (Fig. 13b). The damping coefficients of the MR dampers can be changed by issuing a command voltage between $0 \mathrm{~V}$ to $1.2 \mathrm{~V}$. This command voltage determines the electric current of the electromagnetic coil inside the MR damper, which, in turn, generates a magnetic field that sets the viscous damping properties of the MR damper fluid (Lin, et al. 2005). Two control systems, the wireless control system and a traditional wire-based control system, are installed in the test structure. For the wireless system, a total of four wireless sensors are installed to measure floor velocities (Fig. 13). Velocity feedback control algorithms presented in a previous paper are used by both the wired and the wireless control systems (Wang, et al. 2007b). In a centralized feedback pattern, real-time data from all sensors are required for making the control decisions for every MR damper. For this test structure, the wire-based system can achieve a sampling rate of $200 \mathrm{~Hz}$; as shown in Fig. 14, the wireless system can achieve a sampling rate of $12.5 \mathrm{~Hz}$.

In order to ensure that appropriate control decisions are computed by the wireless control units, one necessary condition is that the real-time velocity data used by the control units are reliable. Rarely experiencing data losses during the experiments, our prototype wireless sensor network proves to be robust. As reported by Lynch, et al. (2008), data losses less than $2 \%$ are experienced. Should data loss be encountered, the wireless control unit is currently designed to simply use the data sample from the previous time step. To illustrate the reliability of the velocity data collected and transmitted by the wireless units, Fig. 15(a) presents the Floor-1 time history data during a centralized wireless control test. The data is 
collected by both the wired DAQ system and the three wireless control units. During the test, unit $C_{1}$ measures the data from the associated velocity meter directly, stores the data in its own memory bank, and transfers the data wirelessly to units $C_{0}$ and $C_{2}$. After the test run is completed, data from all the three control units are sequentially streamed to the experiment command server, where the results are plotted as shown in Fig. 15(a). These plots illustrate strong agreement among data recorded by the three wireless control units and by the wired system using a separate set of velocity meters and data acquisition system. It is shown that the velocity data are not only reliably measured by unit $C_{0}$, but also properly transmitted to other wireless control units in real-time.
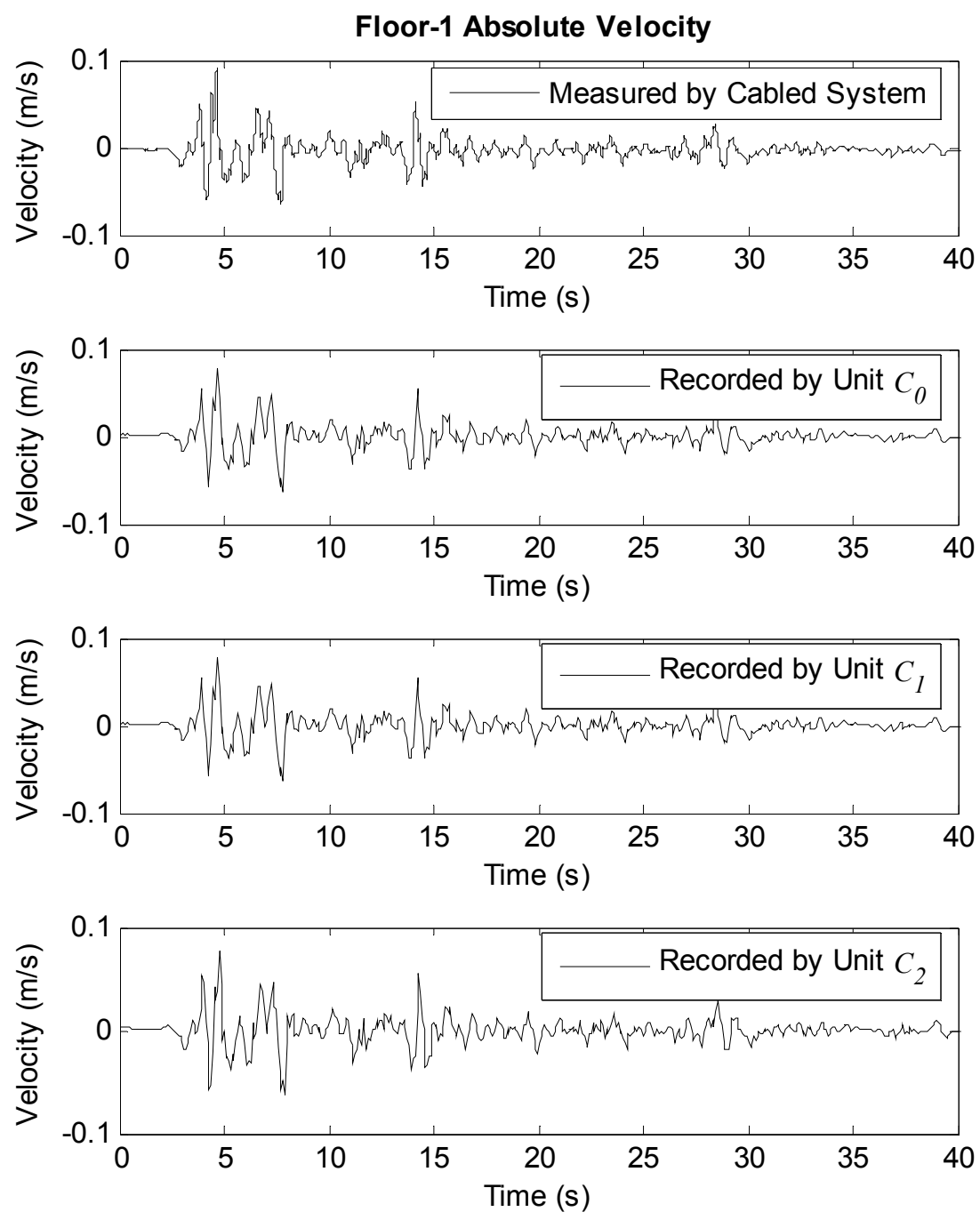

(a) Floor-1 absolute velocity data recorded by the cabled and wireless sensing systems 
Floor $3 / 2$ Inter-Story Drift under El Centro Excitation (Peak $1 \mathrm{~m} / \mathrm{s}^{2}$ )

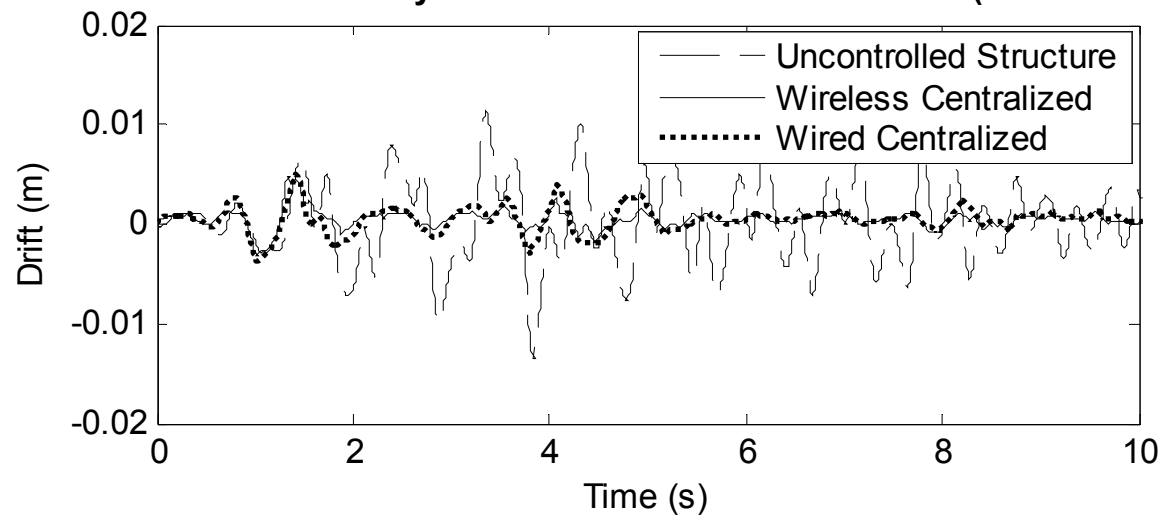

Floor 2/1 Inter-Story Drift under El Centro Excitation (Peak $1 \mathrm{~m} / \mathrm{s}^{2}$ )

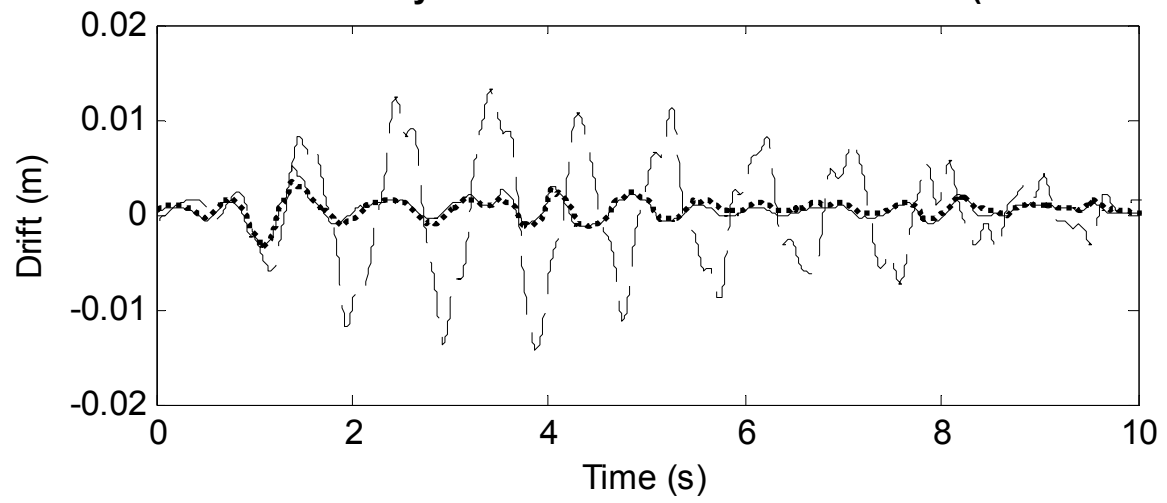

Floor $1 / 0$ Inter-Story Drift under El Centro Excitation (Peak $1 \mathrm{~m} / \mathrm{s}^{2}$ )

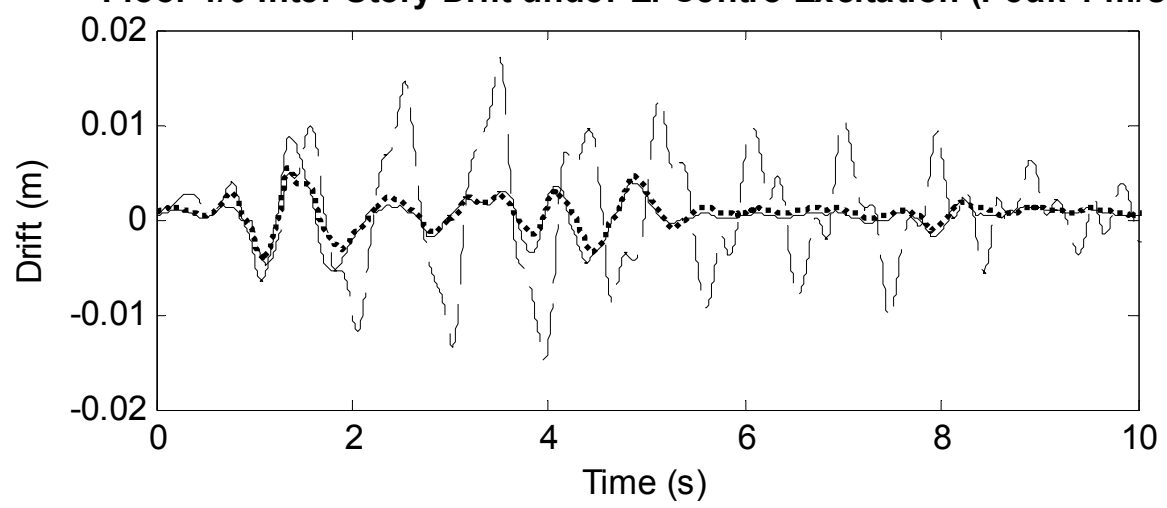

(b) Inter-story drifts of the structure with and without control

Fig. 15. Experimental time histories 
The time histories of the inter-story drifts from the same centralized wireless control test are plotted in Fig. 15(b), together with the drifts of a centralized wired control test and a bare structure test when the structure is not instrumented with any control system (i.e. the MR dampers are not installed). The same ground excitation (1940 El Centro NS earthquake record scaled to a peak ground acceleration of $\left.1 \mathrm{~m} / \mathrm{s}^{2}\right)$ is used for all the three cases shown in Fig. 15(b). The results show that both the wireless and wired control systems achieve considerable performance in mitigating inter-story drifts. Running at a much shorter sampling time step, the wired centralized control system achieves slightly better control performance than the wireless centralized system in terms of mitigating inter-story drifts.

To further study different decentralized schemes with different communication latencies, three wireless control architectures are compared: (\#1) decentralized, (\#2) partially decentralized, and (\#3) centralized. Fig. 16 illustrates the information feedback pattern of each control architecture. The fully decentralized pattern (Wireless \#1) specifies that when computing control decisions, the MR damper at each floor only needs the inter-story velocity difference at Story 1 . The partially decentralized pattern (Wireless \#2) specifies that the control decisions require inter-story velocity from a neighboring floor. Finally, the centralized pattern (Wireless \#3) indicates all velocities relative to ground are required by the control decisions. Different information patterns result in different sampling frequencies for each control architecture. Compared with the centralized scheme, the advantage of a decentralized architecture is that fewer communication and data processing are needed at each sampling time step, thereby reducing sampling time step length. As shown in Fig. 16, the wireless system can achieve a sampling rate of $16.67 \mathrm{~Hz}$ for partially decentralized control and $50 \mathrm{~Hz}$ for fully decentralized control.

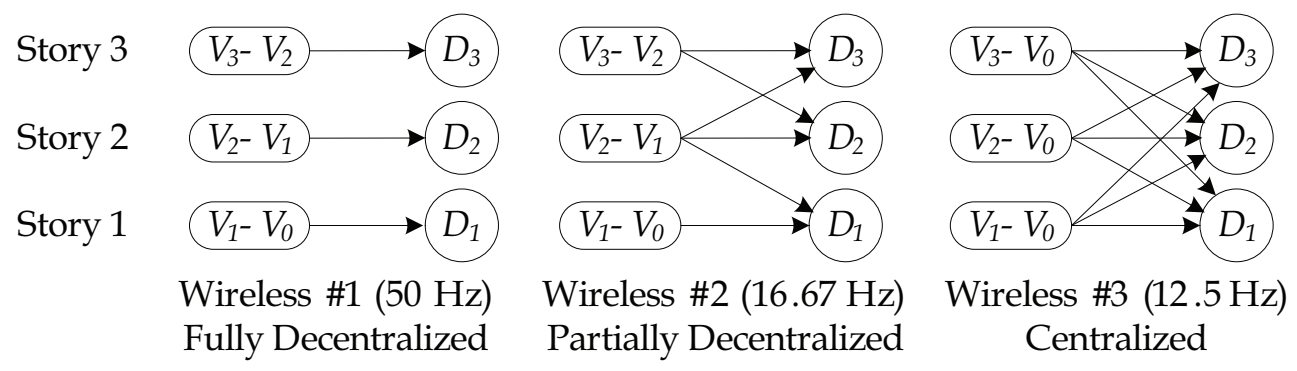

Fig. 16. Various decentralized and centralized information feedback

Fig. 17 shows the peak inter-story drifts and floor accelerations for the original uncontrolled structure and the structure controlled by the three different wireless schemes, as well as the wired centralized control scheme. The $1940 \mathrm{El}$ Centro NS record is employed as the ground excitation, with peak ground acceleration scaled to $1 \mathrm{~m} / \mathrm{s}^{2}$. Compared with the uncontrolled structure, all wireless and wired control schemes achieve significant reduction with respect to maximum inter-story drifts and absolute accelerations. Among the four control cases, the wired centralized control scheme shows good performance in mitigating both peak drifts and peak accelerations. This result is expected because the wired system has the advantages of lower communication latency and utilizes sensor data from all floors. The wireless schemes, although running at longer sampling steps, achieve control performance comparable to the wired system. For all three earthquake records, the fully decentralized wireless 
control scheme (Wireless \#1) results in low peak inter-story drifts and the smallest peak floor accelerations at most of the floors. This result illustrates that in the decentralized wireless control cases, the higher sampling rate (achieved due to lower communication latency) potentially compensates for the lack of data from faraway floors.
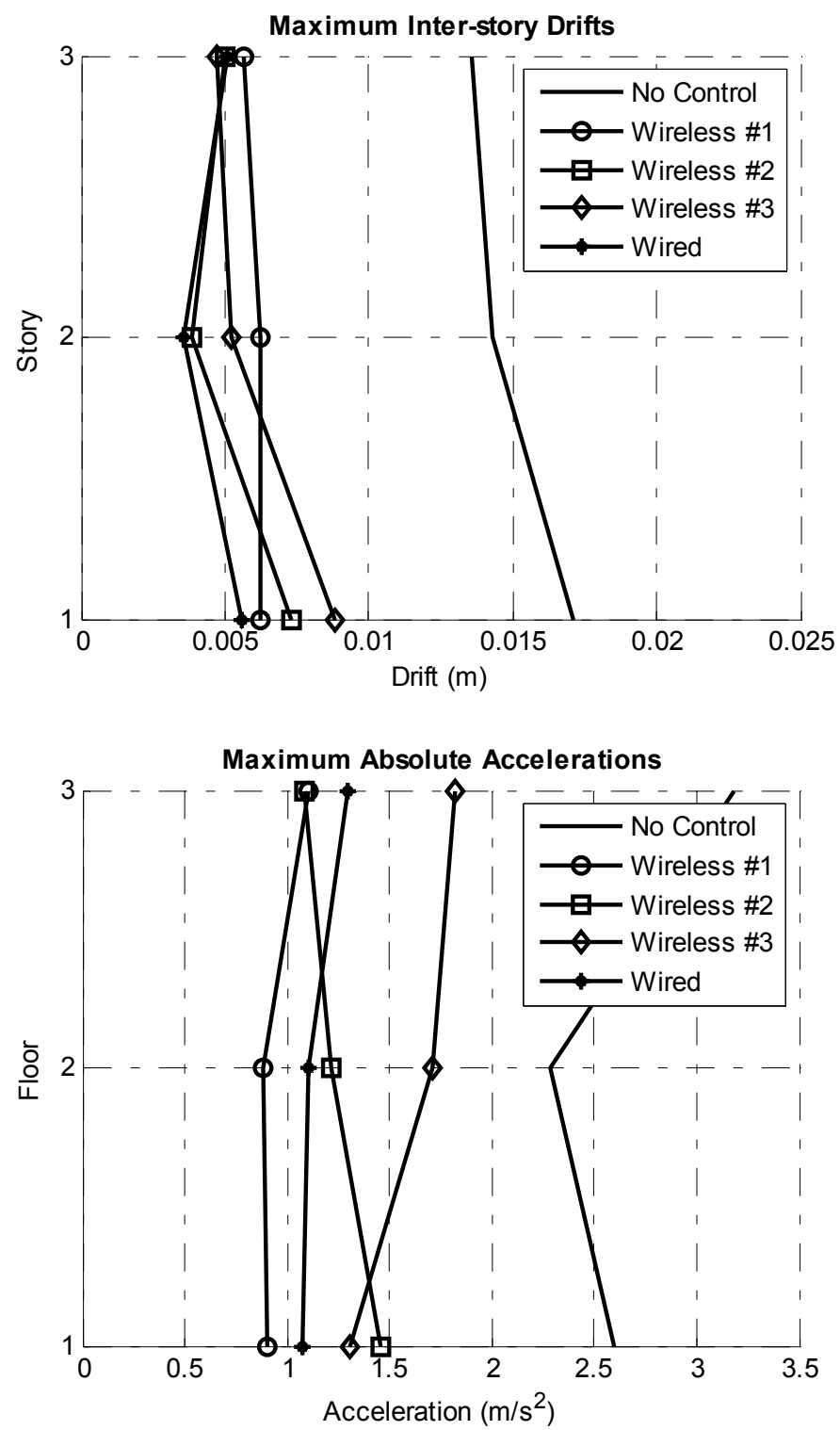

Fig. 17. Experimental results of different control schemes under 1940 El Centro NS earthquake excitation with peak ground accelerations (PGA) scaled to $1 \mathrm{~m} / \mathrm{s}^{2}$ 


\section{Summary and discussion}

This chapter discusses the various issues of applying wireless sensor networks to modern smart structural technologies, including structural health monitoring and structural control. Autonomous wireless sensing and control units with embedded computing can serve as the building blocks of a smart structural system. For different structural applications, design concepts have been proposed to address the information constraints in a wireless sensor network, such as bandwidth, latency, range, and reliability. Robust communication protocol design for centralized and decentralized information architectures is proposed for efficiently managing the information flow in the wireless network. State machine concepts prove to be effective in designing simple yet efficient communication protocols for wireless structural sensing and control networks. Large-scale laboratory and field validation tests have been conducted to validate the efficacy and robustness of the information management schemes implemented in the wireless structural monitoring and control system. Most recently, the prototype wireless sensing system has been successfully tested for long-range measurement of low-amplitude and low-frequency vibrations at Canton Tower, a.k.a. Guangzhou TV and Sightseeing Tower, the world's tallest TV tower upon construction (Ni, et al. 2011).

A common trend in both structural monitoring and structural control application is the increasingly dense deployment of system nodes, i.e. sensors in a structural monitoring system, or sensors, controllers, and control devices in a structural control system. For example, in structural monitoring systems for cable-supported bridges, hundreds of sensors are often deployed for recording loading conditions and bridge responses (Wong 2004, Ko and Ni 2005, Çelebi 2006). Among many modern structural control systems, hundreds of semi-active hydraulic dampers have been installed in high-rise buildings (Kurino, et al. 2003, Spencer and Nagarajaiah 2003, Shimizu, et al. 2004). With rapid advancement in wireless sensor networks, there will be an inevitable trend of reduced system cost yet increased system nodal densities. Particularly in recent years, more and more large-scale wireless structural health monitoring (Lynch, et al. 2006, Kim, et al. 2007, Weng, et al. 2008, Whelan and Janoyan 2009, Rice, et al. 2010) and wireless structural control (Swartz and Lynch 2009, Wang and Law 2011) studies have been reported. Furthermore, researchers have started interesting exploration on mobile sensor networks, as the next-generation wireless sensor networks, for structural health monitoring applications (Zhu, et al. 2010). Such a mobile sensor network involves miniature autonomous mobile robots that carry wireless sensors and automatically move upon a large structure. In summary, it is believed that future monitoring and control systems will enjoy tremendous opportunities provided by the continuing advancements in wireless sensor technologies.

\section{Acknowledgment}

This research was partially funded by the National Science Foundation under grants CMS9988909 and CMMI-0824977 (Stanford University), as well as CMMI-0928095 (Georgia Institute of Technology). The first author was supported by an Office of Technology Licensing Stanford Graduate Fellowship. Prof. Jerome P. Lynch at University of Michigan kindly provided insightful advices to the development of the prototype wireless sensing and control system. Prof. Chin-Hsiung Loh, Dr. Pei-Yang Lin, and Dr. Kung-Chun Lu at the National Taiwan University offered generous support for conducting the shake table experiments at NCREE, Taiwan. The authors would also like to express their gratitude to 
Prof. Ahmed Elgamal and Dr. Michael Fraser of the University of California, San Diego, for their generous assistance throughout the field validation tests at Voigt Bridge.

\section{References}

Çelebi, M. (2006). Real-time seismic monitoring of the new Cape Girardeau Bridge and preliminary analyses of recorded data: an overview. Earthquake Spectra, Vol. 22, No. 3, pp. 609-630

Çelebi, M. (2002). Seismic Instrumentation of Buildings (with Emphasis on Federal Buildings). Report No. 0-7460-68170, United States Geological Survey, Menlo Park, CA

Chang, F.-K. (Ed.) Structural Health Monitoring 2009: From System Integration to Autonomous Systems, Proceedings of the 6th International Workshop on Structural Health Monitoring, Lancaster, PA, USA, September 9-11, 2009

Cooklev, T. (2004). Wireless Communication Standards : a Study of IEEE 802.11, 802.15, and 802.16, Standards Information Network IEEE Press, New York

Doebling, S. W., Farrar, C. R. \& Cornwell, P. J. (1997). DIAMOND: A graphical interface toolbox for comparative modal analysis and damage identification, Proceedings of the 6th International Conference on Recent Advances in Structural Dynamics, Southampton, UK, July 14 - 17, 1997

Farrar, C. R., Sohn, H., Hemez, F. M., Anderson, M. C., Bement, M. T., Cornwell, P. J., Doebling, S. W., Schultze, J. F., Lieven, N. \& Robertson, A. N. (2003). Damage Prognosis: Current Status and Future Needs. Report No. LA-14051-MS, Los Alamos National Laboratory, Los Alamos, NM

Fraser, M., Elgamal, A. \& Conte, J. P. (2006). UCSD Powell Laboratory Smart Bridge Testbed. Report No. SSRP 06/06, Department of Structural Engineering, University of California, San Diego, La Jolla, CA

Housner, G. W., Bergman, L. A., Caughey, T. K., Chassiakos, A. G., Claus, R. O., Masri, S. F., Skelton, R. E., Soong, T. T., Spencer, B. F., Jr. \& Yao, J. T. P. (1997). Structural control: past, present, and future. Journal of Engineering Mechanics, Vol. 123, No. 9, pp. 897-971

Janssen, G. J. M. \& Prasad, R. (1992). Propagation measurements in an indoor radio environment at $2.4 \mathrm{GHz}, 4.75 \mathrm{GHz}$ and $11.5 \mathrm{GHz}$, Proceedings of IEEE 42nd Vehicular Technology Conference, Denver, CO, May 10 - 13, 1992

Kim, S., Pakzad, S., Culler, D., Demmel, J., Fenves, G., Glaser, S. \& Turon, M. (2007). Health monitoring of civil infrastructures using wireless sensor networks, Proceedings of the 6th International Conference on Information Processing in Sensor Networks (IPSN '07), Cambridge, MA, April 25 - 27, 2007

Ko, J. M. \& Ni, Y. Q. (2005). Technology developments in structural health monitoring of large-scale bridges. Engineering Structures, Vol. 27, No. 12, pp. 1715-1725

Kurino, H., Tagami, J., Shimizu, K. \& Kobori, T. (2003). Switching oil damper with built-in controller for structural control. Journal of Structural Engineering, Vol. 129, No. 7, pp. 895-904

Lin, P.-Y., Roschke, P. N. \& Loh, C.-H. (2005). System identification and real application of the smart magneto-rheological damper, Proceedings of the 2005 International Symposium on Intelligent Control, Limassol, Cyprus, June 27 - 29, 2005

Lynch, J. P. \& Loh, K. J. (2006). A summary review of wireless sensors and sensor networks for structural health monitoring. The Shock and Vibration Digest, Vol. 38, No. 2, pp. 91-128 
Lynch, J. P., Wang, Y., Loh, K. J., Yi, J.-H. \& Yun, C.-B. (2006). Performance monitoring of the Geumdang Bridge using a dense network of high-resolution wireless sensors. Smart Materials and Structures, Vol. 15, No. 6, pp. 1561-1575

Lynch, J. P., Wang, Y., Swartz, R. A., Lu, K.-C. \& Loh, C.-H. (2008). Implementation of a closed-loop structural control system using wireless sensor networks. Structural Control and Health Monitoring, Vol. 15, No. 4, pp. 518-539

MaxStream, Inc. (2004). 9XCite ${ }^{\mathrm{TM}}$ OEM RF Module Product Manual v1.1. Lindon, UT

MaxStream, Inc. (2005). XStream ${ }^{\mathrm{TM}}$ OEM RF Module Product Manual v4.2B. Lindon, UT

Molisch, A. F. (2005). Wireless Communications, John Wiley \& Sons, IEEE Press, Chichester, West Sussex, England

Ni, Y. Q., Li, B., Lam, K. H., Zhu, D., Wang, Y., Lynch, J. P. \& Law, K. H. (2011). Inconstruction vibration monitoring of a super-tall structure using a long-range wireless sensing system. Smart Structures and Systems, Vol. 7, No. 2, pp. 83-102

Rappaport, T. S. \& Sandhu, S. (1994). Radio-wave propagation for emerging wireless personal-communication systems. Antennas and Propagation Magazine, IEEE, Vol. 36, No. 5, pp. 14-24

Rice, J. A., Mechitov, K., Sim, S.-H., Nagayama, T., Jang, S., Kim, R., B. F. Spencer, J., Agha, G. \& Fujino, Y. (2010). Flexible smart sensor framework for autonomous structural health monitoring. Smart Structures and Systems, Vol. 6, No. 5, pp. 423-438

Richardson, M. H. (1997). Is it a mode shape, or an operating deflection shape? Sound and Vibration Magazine, Vol. 31, No. pp. 54-61

Shimizu, K., Yamada, T., Tagami, J. \& Kurino, H. (2004). Vibration tests of actual buildings with semi-active switching oil damper, Proceedings of the 13th World Conference on Earthquake Engineering, Vancouver, B.C., Canada, August 1 - 6, 2004

Sohn, H., Farrar, C. R., Hemez, F. M., Shunk, D. D., Stinemates, D. W. \& Nadler, B. R. (2003). A Review of Structural Health Monitoring Literature: 1996-2001. Report No. LA-13976MS, Los Alamos National Laboratory, Los Alamos, NM

Soong, T. T. (1990). Active Structural Control: Theory and Practice, Wiley, Harlow, Essex, England

Spencer, B. F., Jr. \& Nagarajaiah, S. (2003). State of the art of structural control. Journal of Structural Engineering, Vol. 129, No. 7, pp. 845-856

Straser, E. G. \& Kiremidjian, A. S. (1998). A Modular, Wireless Damage Monitoring System for Structures. Report No. 128, John A. Blume Earthquake Eng. Ctr., Stanford University, Stanford, CA

Swartz, R. A. \& Lynch, J. P. (2009). Strategic network utilization in a wireless structural control system for seismically excited structures. Journal of Structural Engineering, Vol. 135, No. 5, pp. 597-608

Tweed, D. (1994). Designing real-time embedded software using state-machine concepts, Circuit Cellar Ink, (53), pp. 12-19.

Wang, Y., Lynch, J. P. \& Law, K. H. (2005). Design of a low-power wireless structural monitoring system for collaborative computational algorithms, Proceedings of SPIE, Health Monitoring and Smart Nondestructive Evaluation of Structural and Biological Systems IV, San Diego, CA, March 9, 2005

Wang, Y. (2007). Wireless Sensing and Decentralized Control for Civil Structures: Theory and Implementation. PhD Thesis, Department of Civil and Environmental Engineering, Stanford University, Stanford, CA 
Wang, Y., Lynch, J. P. \& Law, K. H. (2007a). A wireless structural health monitoring system with multithreaded sensing devices: design and validation. Structure and Infrastructure Engineering, Vol. 3, No. 2, pp. 103-120

Wang, Y., Swartz, R. A., Lynch, J. P., Law, K. H., Lu, K.-C. \& Loh, C.-H. (2007b). Decentralized civil structural control using real-time wireless sensing and embedded computing. Smart Structures and Systems, Vol. 3, No. 3, pp. 321-340

Wang, Y. \& Law, K. H. (2011). Structural control with multi-subnet wireless sensing feedback: experimental validation of time-delayed decentralized $\mathrm{H}_{\infty}$ control design. Advances in Structural Engineering, Vol. 14, No. 1, pp. 25-39

Weng, J.-H., Loh, C.-H., Lynch, J. P., Lu, K.-C., Lin, P.-Y. \& Wang, Y. (2008). Output-only modal identification of a cable-stayed bridge using wireless monitoring systems. Engineering Structures, Vol. 30, No. 7, pp. 1820-1830

Whelan, M. J. \& Janoyan, K. D. (2009). Design of a robust, high-rate wireless sensor network for static and dynamic structural monitoring. Journal of Intelligent Material Systems and Structures, Vol. 20, No. 7, pp. 849-863

Wong, K.-Y. (2004). Instrumentation and health monitoring of cable-supported bridges. Structural Control and Health Monitoring, Vol. 11, No. 2, pp. 91-124

Yao, J. T. P. (1972). Concept of structural control. Journal of Structural Division, ASCE, Vol. 98, No. 7, pp. 1567-1574

Zhu, D., Yi, X., Wang, Y., Lee, K.-M. \& Guo, J. (2010). A mobile sensing system for structural health monitoring: design and validation. Smart Materials and Structures, Vol. 19, No. 5, pp. 055011 


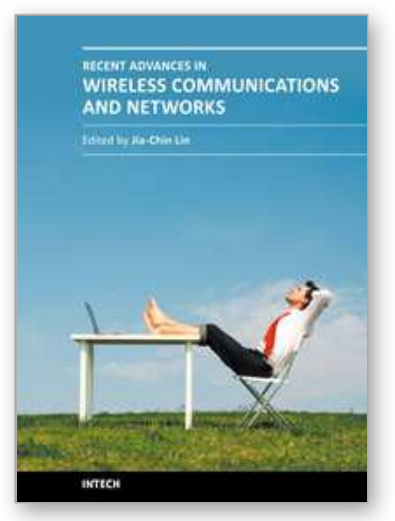

\author{
Recent Advances in Wireless Communications and Networks \\ Edited by Prof. Jia-Chin Lin
}

ISBN 978-953-307-274-6

Hard cover, 454 pages

Publisher InTech

Published online 23, August, 2011

Published in print edition August, 2011

This book focuses on the current hottest issues from the lowest layers to the upper layers of wireless communication networks and provides â€œreal-timeâ€ research progress on these issues. The authors have made every effort to systematically organize the information on these topics to make it easily accessible to readers of any level. This book also maintains the balance between current research results and their theoretical support. In this book, a variety of novel techniques in wireless communications and networks are investigated. The authors attempt to present these topics in detail. Insightful and reader-friendly descriptions are presented to nourish readers of any level, from practicing and knowledgeable communication engineers to beginning or professional researchers. All interested readers can easily find noteworthy materials in much greater detail than in previous publications and in the references cited in these chapters.

\title{
How to reference
}

In order to correctly reference this scholarly work, feel free to copy and paste the following:

Yang Wang and Kincho H. Law (2011). Wireless Sensor Networks in Smart Structural Technologies, Recent Advances in Wireless Communications and Networks, Prof. Jia-Chin Lin (Ed.), ISBN: 978-953-307-274-6, InTech, Available from: http://www.intechopen.com/books/recent-advances-in-wireless-communications-andnetworks/wireless-sensor-networks-in-smart-structural-technologies

\section{INTECH}

open science | open minds

\author{
InTech Europe \\ University Campus STeP Ri \\ Slavka Krautzeka 83/A \\ 51000 Rijeka, Croatia \\ Phone: +385 (51) 770447 \\ Fax: +385 (51) 686166 \\ www.intechopen.com
}

\author{
InTech China \\ Unit 405, Office Block, Hotel Equatorial Shanghai \\ No.65, Yan An Road (West), Shanghai, 200040, China \\ 中国上海市延安西路65号上海国际贵都大饭店办公楼 405 单元 \\ Phone: +86-21-62489820 \\ Fax: +86-21-62489821
}


(C) 2011 The Author(s). Licensee IntechOpen. This chapter is distributed under the terms of the Creative Commons Attribution-NonCommercialShareAlike-3.0 License, which permits use, distribution and reproduction for non-commercial purposes, provided the original is properly cited and derivative works building on this content are distributed under the same license. 